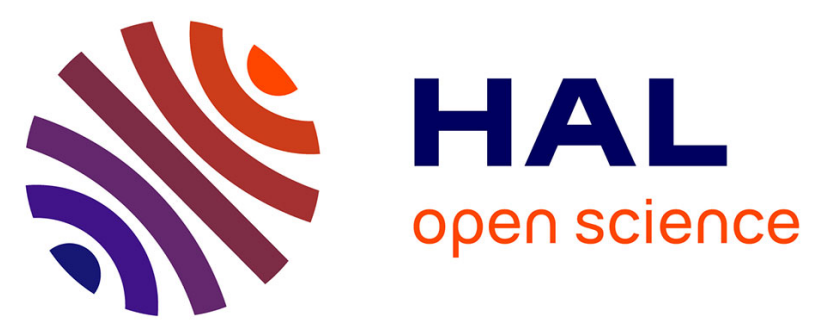

\title{
Numerical analysis of a nonlinearly stable and positive Control Volume Finite Element scheme for Richards equation with anisotropy
}

Ahmed Ait Hammou Oulhaj, Clément Cancès, Claire Chainais-Hillairet

\section{To cite this version:}

Ahmed Ait Hammou Oulhaj, Clément Cancès, Claire Chainais-Hillairet. Numerical analysis of a nonlinearly stable and positive Control Volume Finite Element scheme for Richards equation with anisotropy. ESAIM: Mathematical Modelling and Numerical Analysis, 2018, 52 (4), pp.1532-1567. $10.1051 / \mathrm{m} 2 \mathrm{an} / 2017012$. hal-01372954

\section{HAL Id: hal-01372954 \\ https://hal.science/hal-01372954}

Submitted on 27 Sep 2016

HAL is a multi-disciplinary open access archive for the deposit and dissemination of scientific research documents, whether they are published or not. The documents may come from teaching and research institutions in France or abroad, or from public or private research centers.
L'archive ouverte pluridisciplinaire HAL, est destinée au dépôt et à la diffusion de documents scientifiques de niveau recherche, publiés ou non, émanant des établissements d'enseignement et de recherche français ou étrangers, des laboratoires publics ou privés. 


\title{
NUMERICAL ANALYSIS OF A NONLINEARLY STABLE AND POSITIVE CONTROL VOLUME FINITE ELEMENT SCHEME FOR RICHARDS EQUATION WITH ANISOTROPY
}

\author{
AHMED AIT HAMMOU OULHAJ, CLÉMENT CANCÈS, \\ AND CLAIRE CHAINAIS-HILLAIRET
}

\begin{abstract}
We extend the nonlinear Control Volume Finite Element scheme of [C. Cancès and C. Guichard, Math. Comp., 85(298):549-580, 2016] to the discretization of Richards equation. This scheme ensures the preservation of the physical bounds without any restriction on the mesh and on the anisotropy tensor. Moreover, it does not require the introduction of the so-called Kirchhoff transform in its definition. It also provides a control on the capillary energy. Based on this nonlinear stability property, we show that the scheme converges towards the unique solution to Richards equation when the discretization parameters tend to 0 . Finally we present some numerical experiments to illustrate the behavior of the method.
\end{abstract}

Keywords. Unsaturated porous media flow, Richards equation, nonlinear discretization, nonlinear stability, convergence analysis

AMS subjects classification. 65M12, 65M08, 76S05

\section{Introduction}

1.1. Presentation of the continuous problem. We are interested in the numerical approximation of Richards equation. It is a degenerate nonlinear parabolic equation modeling unsaturated flow in porous media. The diffusion terms can be anisotropic and heterogeneous. In order to ease the reading, we restrict our study to the case of a two-dimensional porous medium. However, the extension of our purpose to the three-dimensional framework does not lead to any theoretical difficulty.

Let $\Omega$ be a polygonal connected open bounded subset of $\mathbb{R}^{2}$, and $t_{f}>0$ a finite time horizon. We define $Q_{t_{f}}=\Omega \times\left(0, t_{f}\right)$. The Richards equation writes:

$$
\begin{cases}\partial_{t} s(p)-\nabla \cdot(\eta(s(p)) \Lambda(\nabla p-\rho \mathbf{g})=0 & \text { in } Q_{t_{f},} \\ s(p)_{t=0}=s_{0} & \text { in } \Omega, \\ \eta(s(p)) \Lambda(\nabla p-\rho \mathbf{g}) \cdot \mathbf{n}=0 & \text { on } \partial \Omega \times\left(0, t_{f}\right) .\end{cases}
$$

In (1), $p$ denotes the water pressure, $s$ the water content, $\eta$ the water mobility function, $\Lambda$ the intrinsic permeability tensor, and $\mathbf{g}$ is the gravity. We do the following assumptions on the data of the continuous problem (1):

(A1) The function $s: \mathbb{R} \rightarrow[0,1]$ is increasing on $\mathbb{R}_{-}$and takes the value 1 on $\mathbb{R}_{+}$. We assume that there exists $p_{\star} \in[-\infty, 0)$ such that $s\left(p_{\star}\right)=0$, and that $s \in L^{1}\left(p_{\star}, 0\right)$. Figure 1 shows two typical profiles of the function $s$.

This work was supported by the GeoPor project funded by the French National Research Agency (ANR) with the grant ANR-13-JS01-0007-01 (project GEOPOR) . 

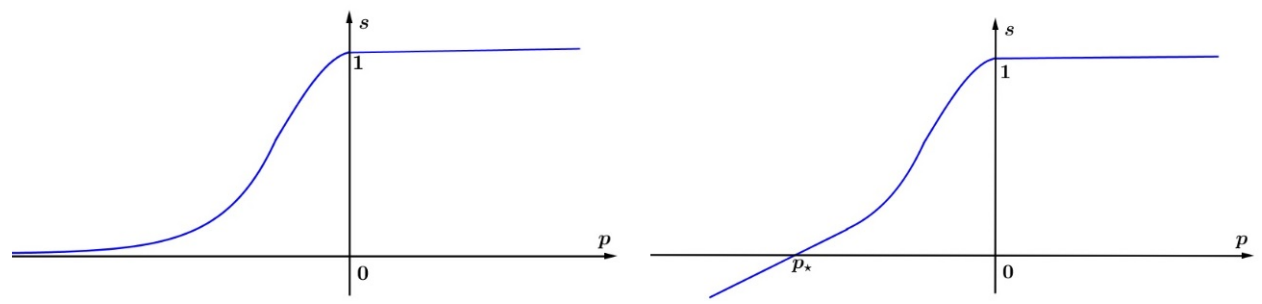

Figure 1. Typical water content functions. Two distinct behaviors are allowed in our study: (on the left) either the function $s$ remains strictly positive on $\mathbb{R}$ but tends to 0 as $p$ tends to $-\infty$ and $p_{\star}=-\infty$, or (on the right) there exists a finite value of $p_{\star}$ such that $s\left(p_{\star}\right)=0$ (right) and the function $s$ is extended into a continuous increasing function on $\left(-\infty, p_{\star}\right]$

(A2) The mobility function $\eta:[0,1] \longrightarrow \mathbb{R}^{+}$is assumed to be continuous, nondecreasing, and to fulfill

$$
\eta(0)=0 \text { and } \eta(s)>0 \text { if } s \neq 0 .
$$

Moreover, we assume all along in this paper that

$$
\xi_{\star}:=\int_{p_{\star}}^{0} \sqrt{\eta(s(a))} \mathrm{d} a<+\infty, \quad \text { and } \quad p^{2} \eta(s(p)) \rightarrow 0, p \rightarrow-\infty .
$$

(A3) The permeability tensor $\Lambda$ belongs $\left(L^{\infty}(\Omega)\right)^{2 \times 2}$, and it is supposed to be symmetric and uniformly elliptic on $\Omega$, i.e, there exists $(\bar{\Lambda}, \underline{\Lambda}) \in \mathbb{R}_{+}^{*} \times \mathbb{R}_{+}^{*}$ such that

$$
\underline{\Lambda}|\mathbf{v}|^{2} \leq \Lambda(\mathbf{x}) \mathbf{v} \cdot \mathbf{v} \leq \bar{\Lambda}|\mathbf{v}|^{2}, \quad \forall \mathbf{v} \in \mathbb{R}^{2}, \quad \text { for a.e. } \quad \mathbf{x} \in \Omega .
$$

(A4) The initial data $s_{0}$ is supposed to belong to $L^{\infty}(\Omega ;[0,1])$, and we assume

$$
0<\bar{s}_{0}:=\frac{1}{\operatorname{meas}(\Omega)} \int_{\Omega} s_{0}(x) \mathrm{d} x<1
$$

Since $s$ is continuous and increasing on $\left[p_{\star}, 0\right]$, there exists a continuous and increasing function $s^{-1}:[0,1] \rightarrow\left[p_{\star}, 0\right]$ such that, $s \circ s^{-1}(\zeta)=\zeta$ for all $\zeta \in[0,1]$. Simple calculations (see for instance [11]) show that

$$
\left\|s^{-1}\right\|_{L^{1}(0,1)}=\|s\|_{L^{1}\left(p_{\star}, 0\right)} \leq C .
$$

thanks to (A1). We define the function $\Gamma: \mathbb{R} \rightarrow \mathbb{R}_{+}$(called capillary energy function) by

$$
\Gamma(p)=\int_{0}^{p} a s^{\prime}(a) \mathrm{d} a .
$$

The function $\Gamma \circ s^{-1}$ is convex on $[0,1]$, and it follows from the definition (6) that

$$
\partial_{t} \Gamma(p)=p \partial_{t} s(p) \text {. }
$$

In order to give a proper mathematical sense to the solution of (1), we need to introduce the Lipschitz continuous increasing function $\xi: \mathbb{R} \longrightarrow \mathbb{R}$ defined by

$$
\xi(p)=\int_{0}^{p} \sqrt{\eta(s(a))} \mathrm{d} a, \quad \forall p \in \mathbb{R} .
$$


We introduce the so-called hydraulic head $u$ defined by

$$
u(\boldsymbol{x}, t)=\frac{p(\boldsymbol{x}, t)}{\rho g}+z(\boldsymbol{x}) \quad \text { for all } \quad(\boldsymbol{x}, t) \in Q_{t_{f}}, \quad \text { for all } \quad t_{f}>0,
$$

where $g$ denotes the modulus of $\mathbf{g}$ and the function $z(\boldsymbol{x})$ is the projection of the point $\boldsymbol{x}$ on the vertical axis, oriented upward by $-\mathrm{g} / \mathrm{g}$. With a simple adimensionalization, we can assume that $\rho g=1$. The system (1) then rewrites:

$$
\begin{cases}\partial_{t} s(p)-\nabla \cdot(\eta(s(p)) \Lambda \nabla u)=0 & \text { in } \quad Q_{t_{f}} \\ s(p)_{t=0}=s_{0} & \text { in } \Omega \\ \eta(s(p)) \Lambda \nabla u \cdot \mathbf{n}=0 & \text { on } \partial \Omega \times\left(0, t_{f}\right)\end{cases}
$$

Remark 1.1. In §5.1, we will present a test case without gravity. Stricto sensu, this case is not included in our study. But it corresponds to the simpler case $p=u$ (mainly carried out in [9]) for which our analysis can be straightforwardly adapted.

Multiplying (formally) the equation (9) by $u$ and integrating on $\Omega$ yields the classical energy/dissipation property:

$$
\frac{\mathrm{d}}{\mathrm{d} t} \int_{\Omega}(\Gamma(p)+s(p) z(\boldsymbol{x})) \mathrm{d} \boldsymbol{x}+\int_{\Omega} \eta(s(p)) \Lambda \nabla u \cdot \nabla u \mathrm{~d} \boldsymbol{x}=0, \quad \forall t \in\left(0, t_{f}\right) .
$$

This allows in particular to show that the capillary energy remains bounded and that the function $\xi(p)$ belongs to $L^{2}\left((0, T) ; H^{1}(\Omega)\right)$, i.e.,

$$
\int_{\Omega} \Gamma(s(p(\boldsymbol{x}, t))) \mathrm{d} \boldsymbol{x}+\int_{0}^{t} \int_{\Omega}|\nabla \xi(p(\boldsymbol{x}, \tau))|^{2} \mathrm{~d} \boldsymbol{x} \mathrm{d} \tau \leq C, \quad \forall t \in\left(0, t_{f}\right) .
$$

Definition 1.2 (weak solution). A measurable function $p: Q_{t_{f}} \rightarrow \mathbb{R}$ is said to be a weak solution of (1) if $p \geq p_{\star}$ a.e. in $Q_{t_{f}}$, if $\xi(p)$ belongs to $L^{2}\left(\left(0, t_{f}\right) ; H^{1}(\Omega)\right)$, and if, for all $\psi \in \mathcal{C}_{c}^{\infty}\left(\bar{\Omega} \times\left[0, t_{f}\right)\right)$, one has

$$
\begin{aligned}
& \iint_{Q_{t_{f}}} s(p) \partial_{t} \psi \mathrm{d} \boldsymbol{x} \mathrm{d} t+\int_{\Omega} s_{0} \psi(., 0) \mathrm{d} \boldsymbol{x} \\
& -\iint_{Q_{t_{f}}} \sqrt{\eta(s(p))} \Lambda \nabla \xi(p) \cdot \nabla \psi \mathrm{d} \boldsymbol{x} \mathrm{d} t-\iint_{Q_{t_{f}}} \eta(s(p)) \rho g \Lambda \nabla z \cdot \nabla \psi \mathrm{d} \boldsymbol{x} \mathrm{d} t=0 .
\end{aligned}
$$

The notion of weak solution is motivated by the following theorem.

Theorem 1.3. Under assumptions (A1)-(A4), there exists a unique weak solution to the problem (1) in the sense of Definition 1.2.

The existence of a solution is a by-product of the convergence of the scheme proved in $\S 4$. It can also be obtained by compactness arguments following the program of Alt and Luckhaus [3]. Concerning the uniqueness, since we consider no-flux boundary conditions, we can not directly apply Otto's result [26], where Dirichlet boundary conditions are imposed. However, a slight adaptation of Otto's proof detailed in appendix (cf. Proposition A.4) allows us to extend the uniqueness result to our framework. 
1.2. Goal and positioning of the paper. Because of its broad interest in the environmental studies, the Richards equation [28] has been the purpose of many research papers, especially in the field of numerical analysis. Richards equation is locally conservative and a particular effort was made to preserve this property in most of the contributions.

A conservative Finite Difference scheme has been studied numerically in [30]. However, there is up to our knowledge no convergence proof for the scheme presented in [30]. Moreover, restrictive conditions have to be prescribed on the grid and on the permeability tensor $\Lambda$. The convergence of Two-Point Flux Approximation Finite Volume schemes have been studied in [18] for a scheme that requires the introduction of the Kirchhoff transform, and in [17] for a scheme expressed in physical variables (saturation and pressure), but under the non-physical assumption that the mobility function was not degenerated (i.e., $\eta(s) \geq \eta_{\star}>0$ for all $s$ ). In both [18] and [17], it was moreover required that the porous medium was isotropic (i.e., $\Lambda=\lambda \mathrm{Id}$ ) and that the mesh satisfies the so-called orthogonality condition (see, e.g., [15, Definition 9.1] and [14]) so that the two-point flux approximation is consistent. Since they are naturally locally conservative, Mixed Finite Elements have been widely used for the approximation of Richards equation. Let us for instance mention $[5,27]$ where the authors managed to provide an error estimate. Nevertheless, the schemes studied in [5, 27] rely on the introduction of the Kirchhoff transform. Let us also mention the extension of Multi-Point Flux Approximation Finite Volume schemes to the context of Richards equation in [24, 7]. Note that Mixed Finite Elements and Multi-Point Flux Approximation Finite Volumes may produce over- and undershoots on the saturation. We refer to [13] for a review of the numerous Finite Volume methods developed in the last decades that can be applied to the discretization of Richards equation.

The method we study here was designed on the following specifications:

(a) to handle anisotropic and heterogeneous anisotropy tensors;

(b) to avoid the introduction of non-physical quantities like, e.g., the Kirchhoff transform;

(c) to preserve the physical bounds on the saturation;

(d) to conserve locally the mass of fluid;

(e) to converge towards the solution to the continuous problem (mathematical proof and numerical evidence).

The scheme we propose belongs to the family of the so-called Control Volume Finite Element schemes introduced in the context of porous media flows by Forsyth [19, 20]. Roughly speaking, it consists in an interpretation of Finite Elements with mass lumping as a locally conservative method on dual cells. It was already noticed in [19] that the grid had to fulfill some restrictive condition unless the transmissivities may become negative. It results that the reconstructed numerical flux goes the opposite sense to the physical one. More precisely, the triangular grid has to fulfill a socalled Delaunay condition in the two-dimensional isotropic case $\Lambda=\lambda \mathrm{Id}$. But in the case where $\Lambda$ is a spatially varying full tensor, there is no algorithm up to our knowledge to build a triangulation such that the transmissivity remain nonnegative. As it will be proved in the sequel, the method we propose still converges even in the case where negative transmissivities appear. Our scheme is an extension of the one studied in $[8,9]$. It is based on a suitable upwinding of the mobility (i.e., w.r.t. the numerical flux and not w.r.t. the physical one) that allows to preserve the 
physical bounds (but not the monotonicity as in [20]). Moreover, we show that our method provides a control on the capillary energy and that this control is sufficient to perform a convergence proof based on compactness arguments.

The paper is organized as follows. In Section 2, we introduce the scheme and we state the main results of our paper. Theorem 2.4 states the existence of a solution to scheme which preserves the physical bounds and for which the capillary energy and the energy dissipation are bounded uniformly w.r.t. the grid. Theorem 2.5 states the convergence of a sequence of approximate solutions given by the scheme to the unique weak solution to (9) (its uniqueness is proved in Appendix). In Section 3, we derive a priori estimates on the discrete solution. They allow us to prove in $\S 3.3$ the existence of a discrete solution to the nonlinear system corresponding to the scheme. Section 4 is devoted to the convergence proof of the scheme. This proof is based first on the compactness of the sequence of approximate solutions and then on the identification of the limit. We finally present numerical experiments in Section 5 , which confirm the theoretical results we proved. We take care to fairly present the advantages and the drawbacks of the method from a computational point of view.

\section{The NUMERICAL SCHEME}

\subsection{Discretization of $Q_{t_{f}}$.}

2.1.1. Discretizations of $\Omega$. The CVFE method requires the introduction of two different space discretizations of $\Omega$ : a primal triangular mesh and a dual barycentric mesh.

The primal triangular mesh is denoted by $\mathcal{T}$. It is a conformal triangular discretization of the polygonal domain $\Omega$, consisting in open bounded separated triangles satisfying $\bigcup_{T \in \mathcal{T}} \bar{T}=\bar{\Omega}$. For $T \in \mathcal{T}$, we denote by $\boldsymbol{x}_{T}$ the center of gravity of $T$, by $h_{T}$ the diameter of the triangle $T$, and by $\rho_{T}$ the diameter of the largest ball inscribed in the triangle $T$. Then, we define the mesh diameter $h$ and the mesh regularity $\theta_{\mathcal{T}}$ by

$$
h=\max _{T \in \mathcal{T}} h_{T}, \quad \theta_{\mathcal{T}}=\max _{T \in \mathcal{T}} \frac{h_{T}}{\rho_{T}} .
$$

We denote by $\mathcal{V}$ the set of the vertices of the discretization $\mathcal{T}$, located at positions $\left(\boldsymbol{x}_{K}\right)_{K \in \mathcal{V}}$. The set $\mathcal{E}$ of the edges of $\mathcal{T}$ is made of straight segments $\sigma$ joining two vertices of $\mathcal{V}$. Given $T, T^{\prime} \in \mathcal{T}$, we assume that $\bar{T} \cap \overline{T^{\prime}}$ is either empty, or it is reduced to $\boldsymbol{x}_{K}$ for some $K \in \mathcal{V}$, or it consists in an edge $\sigma$ belonging $\mathcal{E}$. For $T \in \mathcal{T}$, we denote by $\mathcal{E}_{T}$ the set of the edges of $T: \bigcup_{\sigma \in \mathcal{E}_{T}} \bar{\sigma}=\partial T$. We assume that $\mathcal{E}=\bigcup_{T \in \mathcal{T}} \mathcal{E}_{\mathcal{T}}$. Given two vertices $K, L \in \mathcal{V}$ of a triangle $T$, then the edge joining $\boldsymbol{x}_{K}$ and $\boldsymbol{x}_{L}$ is denoted by $\sigma_{K L}$. For $K \in \mathcal{V}$, one denotes by $\mathcal{T}_{K}$ the subset of $\mathcal{T}$ made the triangles admitting $K$ as a vertex, by $\mathcal{E}_{K}$ the set of edges having the vertex $K$ as an extremity, and by $\mathcal{V}_{K}$ the subset of $\mathcal{V}$ such that, if $L \in \mathcal{V}_{K}$, then $\left[\boldsymbol{x}_{K}, \boldsymbol{x}_{L}\right]$ is an edge of $\mathcal{E}_{K}$.

Once the primal triangular mesh has been built, we can define its dual barycentric mesh $\mathcal{M}$ as follows. To each $K \in \mathcal{V}$, we associate a cell $\omega_{K}$ whose vertices are the isobarycenters $\boldsymbol{x}_{T}$ of the triangles $T \in \mathcal{T}_{K}$ and the isobarycenters $\boldsymbol{x}_{\sigma}$ of the edges $\sigma \in \mathcal{E}_{K}$. Note that $\bar{\Omega}=\bigcup_{K \in \mathcal{V}} \bar{\omega}_{K}$. We refer to Figure 2 for an illustration of the primary and dual barycentric meshes. The 2-dimensional Lebesgue measure of $\omega_{K}$ is denoted by $m_{K}$. 
Let us now introduce some useful functional spaces. The space $V_{\mathcal{T}} \subset \mathcal{C}(\bar{\Omega})$ is made of piecewise affine functions on the primal mesh, i.e.,

$$
V_{\mathcal{T}}=\left\{f \in H^{1}(\Omega) \mid f_{\left.\right|_{T}} \text { is affine, } \forall T \in \mathcal{T}\right\} .
$$

For all $K \in \mathcal{V}$, we denote by $e_{K}$ the unique element of $V_{\mathcal{T}}$ such that $e_{K}\left(\boldsymbol{x}_{K}\right)=1$ and $e_{K}\left(\boldsymbol{x}_{L}\right)=0$ if $L \in \mathcal{V} \backslash\{K\}$. The geometrical construction of $\omega_{K}$ ensures that

$$
\int_{\Omega} e_{K}(\boldsymbol{x}) \mathrm{d} \boldsymbol{x}=\int_{\omega_{K}} \mathrm{~d} \boldsymbol{x}=: m_{K}, \quad \forall K \in \mathcal{V} .
$$

We can also define the set of the piecewise constant functions on $\mathcal{M}, X_{\mathcal{M}}$, by

$$
X_{\mathcal{M}}=\left\{f: \Omega \longrightarrow \mathbb{R} \text { measurable } \mid f_{\left.\right|_{\omega_{K}}} \in \mathbb{R} \text { is constant, } \forall K \in \mathcal{V}\right\}
$$

Given a vector $\left(u_{K}\right)_{K \in \mathcal{V}} \in \mathbb{R}^{\# \mathcal{V}}$, there exists a unique $u_{\mathcal{T}} \in V_{\mathcal{T}}$ and a unique $u_{\mathcal{M}} \in X_{\mathcal{M}}$ such that $u_{\mathcal{T}}\left(\boldsymbol{x}_{K}\right)=u_{\mathcal{M}}\left(\boldsymbol{x}_{K}\right)=u_{K}$ for all $K, L \in \mathcal{V}$. Let us note that $u_{\mathcal{T}}=\sum_{K \in \mathcal{V}} u_{K} e_{K}$. Moreover, for all $q \in[1, \infty)$, there exist $C_{1}$ and $C_{2}$ depending only on $q$ and on $\theta_{\mathcal{T}}$ such that

$$
C_{1}\left\|u_{\mathcal{T}}\right\|_{L^{q}(\Omega)} \leq\left\|u_{\mathcal{M}}\right\|_{L^{q}(\Omega)} \leq C_{2}\left\|u_{\mathcal{T}}\right\|_{L^{q}(\Omega)}, \quad \forall\left(u_{K}\right)_{K \in \mathcal{V}} \in \mathbb{R}^{\# \mathcal{V}} .
$$

A proof of the above inequalities can be found for instance in [10, Lemma A.6].

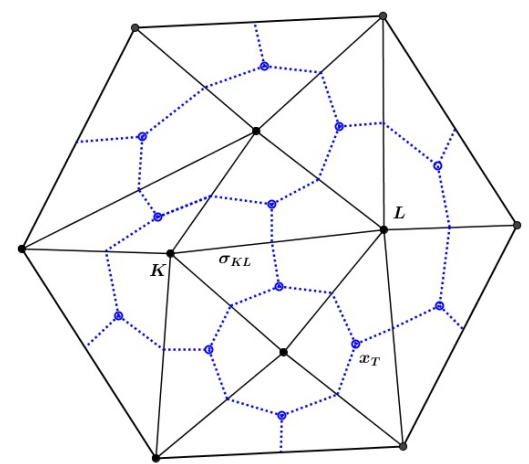

FIgURE 2. The triangular mesh $\mathcal{T}$ (solid line) and its corresponding dual barycentric dual mesh $\mathcal{M}$ (dashed line).

2.1.2. Space-time discretizations. In order to avoid heavier notations, we restrict our study to the case of a uniform time discretization of $\left(0, t_{f}\right)$. However, all the results presented in this paper can be extended to general time discretizations without any technical difficulty. In what follows, we assume that the spatial mesh is fixed and does not change with the time step.

Let $N$ be a nonnegative integer, then we define $\Delta t=\frac{t_{f}}{N+1}$, and $t_{n}=n \Delta t$ for all $n \in\{0, \ldots, N+1\}$, so that $t_{0}=0$, and $t_{N+1}=t_{f}$.

We define the space and time discrete spaces $V_{\mathcal{T}, \Delta t}$ and $X_{\mathcal{M}, \Delta t}$ as the set of piecewise constant functions in time with values in $V_{\mathcal{T}}$ and $X_{\mathcal{M}}$ respectively:

$$
\begin{aligned}
V_{\mathcal{T}, \Delta t} & =\left\{f: Q_{t_{f}} \rightarrow \overline{\mathbb{R}} \mid f(x, t)=f\left(x, t^{n+1}\right) \in V_{\mathcal{T}}, \quad \forall t \in\left(t_{n}, t_{n+1}\right]\right\}, \\
X_{\mathcal{M}, \Delta t} & =\left\{f: Q_{t_{f}} \rightarrow \overline{\mathbb{R}} \mid f(x, t)=f\left(x, t^{n+1}\right) \in X_{\mathcal{M}}, \quad \forall t \in\left(t_{n}, t_{n+1}\right]\right\} .
\end{aligned}
$$


For a given $\left(u_{K}^{n+1}\right)_{n \in\{0, \ldots, N\}, K \in \mathcal{V}} \in \mathbb{R}^{(N+1) \# \mathcal{V}}$, we denote by $u_{\mathcal{T}, \Delta t}$ and $u_{\mathcal{M}, \Delta t}$ the unique elements of $V_{\mathcal{T}, \Delta t}$ and $X_{\mathcal{M}, \Delta t}$ respectively such that

$$
u_{\mathcal{T}, \Delta t}\left(x_{K}, t\right)=u_{\mathcal{M}, \Delta t}\left(x_{K}, t\right)=u_{K}^{n+1}, \quad \forall K \in \mathcal{V}, \forall t \in\left(t_{n}, t_{n+1}\right] .
$$

2.2. Finite elements. The method we propose, and more generally the CVFE method, is based on $P_{1}$-finite elements. We introduce in this section the technical material that is needed in order to define the scheme and to perform its analysis. We define the transmissibility coefficients

$$
a_{K L}^{T}=-\int_{T} \Lambda \nabla e_{K} \cdot \nabla e_{L} \mathrm{~d} \boldsymbol{x}=a_{L K}^{T}, \quad \forall T \in \mathcal{T}, \forall(K, L) \in \mathcal{V}^{2}
$$

and

$$
a_{K L}=a_{L K}=-\int_{\Omega} \Lambda \nabla e_{K} \cdot \nabla e_{L} \mathrm{~d} \boldsymbol{x}=\sum_{T \in \mathcal{T}} a_{K L}^{T}, \quad \forall(K, L) \in \mathcal{V}^{2} .
$$

Note that $a_{K L}=0$ unless $\sigma_{K L} \in \mathcal{E}$. Moreover, since $\sum_{K \in \mathcal{V}} \nabla e_{K}=0$, we have that :

$$
-a_{K K}=\sum_{L \neq K} a_{K L}>0
$$

As a consequence of (16)-(17), given $u_{\mathcal{T}}$ and $v_{\mathcal{T}}$ two elements of $V_{\mathcal{T}}$, one has

$$
\begin{aligned}
\int_{\Omega} \Lambda \nabla u_{\mathcal{T}} \cdot \nabla v_{\mathcal{T}} \mathrm{d} \mathbf{x}=\sum_{\sigma_{K L} \in \mathcal{E}} a_{K L}\left(u_{K}-u_{L}\right)\left(v_{K}-v_{L}\right) \\
=\sum_{T \in \mathcal{T}} \sum_{\sigma_{K L} \in \mathcal{E}_{T}} a_{K L}^{T}\left(u_{K}-u_{L}\right)\left(v_{K}-v_{L}\right) .
\end{aligned}
$$

The following lemma plays a crucial role in the numerical analysis carried out in this paper. We refer to [9, Lemma 3.2] for its proof.

Lemma 2.1. There exists $C_{3}$ depending only on $\theta_{\mathcal{T}}, \Lambda_{\star}$ and $\Lambda^{\star}$ such that, for all $u_{\mathcal{T}} \in V_{\mathcal{T}}$, one has

$$
\sum_{\sigma_{K L} \in \mathcal{E}}\left|a_{K L}\right|\left(u_{K}-u_{L}\right)^{2} \leq \sum_{T \in \mathcal{T}} \sum_{\sigma_{K L} \in \mathcal{E}_{T}}\left|a_{K L}^{T}\right|\left(u_{K}-u_{L}\right)^{2} \leq C_{3} \int_{\Omega} \Lambda \nabla u_{\mathcal{T}} \cdot \nabla u_{\mathcal{T}} \mathrm{d} \boldsymbol{x} .
$$

2.3. The nonlinear CVFE scheme. In this section, we explicit the discretization of the problem (1) we will study in this paper. The time discretization relies on backward Euler scheme, while the space discretization relies on finite elements with mass lumping and a suitable upwinding of the mobility.

The discretization $s_{\mathcal{M}}^{0} \in X_{\mathcal{M}}$ of the initial data is defined by

$$
s_{K}^{0}=\frac{1}{m_{K}} \int_{\omega_{K}} s_{0}(\boldsymbol{x}) \mathrm{d} \boldsymbol{x}, \quad \forall K \in \mathcal{V} .
$$

In the sequel, we will make use of the shortened notation

$$
z_{K}=z\left(\boldsymbol{x}_{K}\right), \quad \forall K \in \mathcal{V} .
$$

Let us now introduce the scheme. For all $n \in\{0, \ldots, N\}$, a solution $\left(p_{K}^{n+1}\right)_{K \in \mathcal{V}}$ to the scheme at the time step $n+1$ has to satisfy the following equations: for all 
$K \in \mathcal{V}$

$$
\begin{aligned}
& \frac{s\left(p_{K}^{n+1}\right)-s_{K}^{n}}{\Delta t} m_{K}+\sum_{\sigma_{K L} \in \mathcal{E}_{K}} \eta_{K L}^{n+1} a_{K L}\left(u_{K}^{n+1}-u_{L}^{n+1}\right)=0 \\
& u_{K}^{n+1}=p_{K}^{n+1}+\rho g z_{K}, \\
& s_{K}^{n+1}=s\left(p_{K}^{n+1}\right), \\
& \eta_{K L}^{n+1}=\left\{\begin{array}{lll}
\eta\left(s_{K}^{n+1}\right) & \text { if } & a_{K L}\left(u_{K}^{n+1}-u_{L}^{n+1}\right) \geq 0 \\
\eta\left(s_{L}^{n+1}\right) & \text { if } & a_{K L}\left(u_{K}^{n+1}-u_{L}^{n+1}\right)<0 .
\end{array}\right.
\end{aligned}
$$

Remark 2.2. It follows from the monotonicity of the mobility and water content functions $\eta$ and $s$ that (20d) is equivalent to

$$
\eta_{K L}^{n+1}=\left\{\begin{array}{ccc}
\max _{p \in I_{K L}^{n+1}} \eta(s(p)) & \text { if } & a_{K L}\left(p_{K}^{n+1}-p_{L}^{n+1}\right)\left(u_{K}^{n+1}-u_{L}^{n+1}\right) \geq 0 \\
\min _{p \in I_{K L}^{n+1}} \eta(s(p)) & \text { if } & a_{K L}\left(p_{K}^{n+1}-p_{L}^{n+1}\right)\left(u_{K}^{n+1}-u_{L}^{n+1}\right) \leq 0
\end{array}\right.
$$

where

$$
I_{K L}^{n+1}=\left[\min \left(p_{K}^{n+1}, p_{L}^{n+1}\right), \max \left(p_{K}^{n+1}, p_{L}^{n+1}\right)\right] .
$$

It is then worth noticing that the monotonicity assumption on $\eta$ can be bypassed if one enforces (21) directly instead of (20d) for the definition of the upwind mobility.

This scheme, whose construction is based on finite elements via (16), can be interpreted as a finite volume scheme. Indeed denoting by

$$
F_{K L}^{n+1}=a_{K L} \eta_{K L}^{n+1}\left(u_{K}^{n+1}-u_{L}^{n+1}\right),
$$

the scheme (20) can be rewritten under the locally conservative form on the dual cells $\omega_{K}$ :

$$
\begin{cases}F_{K L}^{n+1}+F_{L K}^{n+1}=0, & \text { for all } \sigma_{K L} \in \mathcal{E}_{K} \\ \frac{s_{K}^{n+1}-s_{K}^{n}}{\Delta t} m_{K}+\sum_{\sigma_{K L} \in \mathcal{E}_{K}} F_{K L}^{n+1}=0, & \text { for all } K \in \mathcal{V} .\end{cases}
$$

As a straightforward consequence, we can claim that the scheme (20) is globally conservative, i.e.,

$$
\sum_{K \in \mathcal{V}} m_{K} s_{K}^{n+1}=\sum_{K \in \mathcal{V}} m_{K} s_{K}^{n}=\int_{\Omega} s_{0}(\boldsymbol{x}) \mathrm{d} \boldsymbol{x}, \quad \forall n \geq 0 .
$$

Remark 2.3. If $p_{K}^{n+1}=p_{L}^{n+1}=-\infty$ for some $\sigma_{K L} \in \mathcal{E}$ and, $n \geq 0$, we have adopted the convention

$$
\eta_{K L}^{n+1}\left(p_{K}^{n+1}-p_{L}^{n+1}\right)=\eta_{K L}^{n+1}\left(p_{K}^{n+1}-p_{L}^{n+1}\right)^{2}=0,
$$

in accordance with assumption (3). However, as it will appear in Lemma 3.10, the situation where $p_{K}^{n+1}=-\infty$ can only occur if $p_{\star}=-\infty$ and $s_{0}$ is identically equal to 0 , but this situation is prohibited by Assumption (A1). 
2.4. Main results. The scheme (20) amounts to a nonlinear system to be solved at each time step. The existence of a solution to this system is therefore non trivial. The first result we highlight is thus the existence of a solution to the scheme (20) and the stability in terms of the discrete capillary energy.

Theorem 2.4. There exists (at least) one solution $\left(p_{K}^{n+1}\right)_{K \in \mathcal{V}, n \in\{0, \ldots, N\}}$ to the scheme (20a). Moreover, $0 \leq s_{K}^{n} \leq 1$ for all $K \in \mathcal{V}$ and for all $n \in\{0, \ldots, M\}$, and there exists $C$ depending only on $\theta_{\mathcal{T}}, \Lambda, \Omega, t_{f},\|s\|_{L^{1}\left(p_{\star}, 0\right)}$, and $\|\eta\|_{\infty}$ such that

$$
\sup _{n \in\{0, \ldots, N\}} \sum_{K \in \mathcal{V}} m_{K} \Gamma\left(p_{K}^{n+1}\right)+\sum_{n=0}^{N} \Delta t \sum_{\sigma_{K L} \in \mathcal{E}} a_{K L}\left(\xi\left(p_{K}^{n+1}\right)-\xi\left(p_{L}^{n+1}\right)\right)^{2} \leq C .
$$

Once we have the discrete solution $\left(p_{K}^{n+1}\right)_{K \in \mathcal{V}, n \in\{0, \ldots, N\}}$ at hand for all meshes and all time discretizations, then we can study the convergence of the scheme when the discretization parameters tend to 0 . More precisely, consider a sequence $\left(\mathcal{T}_{m}\right)_{m \geq 1}$ of triangulations of $\Omega$ such that

$$
h_{m}=\max _{T \in \mathcal{T}_{m}} \operatorname{diam}(T) \underset{m \rightarrow \infty}{\longrightarrow} 0,
$$

and such that there exists $\theta^{\star}>0$ such that

$$
\theta_{\mathcal{T}_{m}} \leq \theta^{\star}, \quad \forall m \geq 1 .
$$

A sequence of dual meshes $\left(\mathcal{M}_{m}\right)_{m \geq 1}$ corresponding to the triangular meshes $\left(\mathcal{T}_{m}\right)_{m \geq 1}$ is built as in $\S 2.1 .1$. Let $\left(N_{m}\right)_{m \geq 1}$ be an increasing sequence of integers, then we define the corresponding sequence of time steps $\Delta t_{m}=\frac{t_{f}}{N_{m}+1}$ tending to 0 as $m$ tends to $\infty$. To this sequence of discretizations of $Q_{t_{f}}$ corresponds a sequence of solutions $\left(p_{K}^{n+1}\right)_{K \in \mathcal{V}_{m}, n \in\left\{0, \ldots, N_{m}\right\}}$ to the scheme. Thanks to these solutions, we can construct the functions $s_{\mathcal{M}_{m}, \Delta t_{m}} \in X_{\mathcal{M}_{m}, \Delta t_{m}}$ and $\xi_{\mathcal{T}_{m}, \Delta t_{m}} \in V_{\mathcal{T}_{m}, \Delta t_{m}}$ defined by

$$
s_{\mathcal{M}_{m}, \Delta t_{m}}\left(\boldsymbol{x}_{K}, t_{n+1}\right)=s\left(p_{K}^{n+1}\right)=s_{K}^{n+1}, \quad \forall K \in \mathcal{V}_{m}, \forall n \in\left\{0, \ldots, N_{m}\right\}
$$

and

$$
\xi_{\mathcal{T}_{m}, \Delta t_{m}}\left(\boldsymbol{x}_{K}, t_{n+1}\right)=\xi\left(p_{K}^{n+1}\right)=\xi_{K}^{n+1}, \quad \forall K \in \mathcal{V}_{m}, \forall n \in\left\{0, \ldots, N_{m}\right\}
$$

Once these sequences of discrete functions at hand, we can state the second main result of this paper, namely the convergence of the scheme (20).

Theorem 2.5. Let $\left(\mathcal{T}_{m}\right)_{m \geq 1}$ be a sequence conformal triangular discretization of $\Omega$ such that (24) and (25) hold. Let $\left(s_{\mathcal{M}_{m}, \Delta t_{m}}\right)_{m}$ and $\left(\xi_{\mathcal{T}_{m}, \Delta t_{m}}\right)_{m}$ be the functions reconstructed from the solutions $\left(\left(p_{K}^{n+1}\right)_{K, n}\right)_{m}$ to the scheme (20) thanks to formulas (26)-(27). Then

$$
\begin{aligned}
& s_{\mathcal{M}_{m}, \Delta t_{m}} \underset{m \rightarrow+\infty}{\longrightarrow} s(p) \quad \text { a.e in } Q_{t_{f}}, \\
& \xi_{\mathcal{T}_{m}, \Delta t_{m}} \underset{m \rightarrow+\infty}{\longrightarrow} \xi(p) \quad \text { weakly in } L^{2}\left(\left(0, t_{f}\right) ; H^{1}(\Omega)\right) \text { and strongly in } L^{2}\left(Q_{t_{f}}\right),
\end{aligned}
$$

where $p$ is the unique solution to the continuous problem (1).

The proof of Theorem 2.4 is addressed in $\S 3$. The convergence of the scheme towards a weak solution is the purpose of $\S 4$, while the uniqueness of the weak solution is proved in appendix, cf. Proposition A.4. Numerical illustrations are provided in $\S 5$. 


\section{Discrete PROPERTIEs, a priori eStimates AND EXISTENCE}

In this section, we establish a priori estimates, among which the positivity of the saturation and the stability of the capillary energy. These estimates allow to prove the existence of a solution to the nonlinear system (20). They are also keystones in order to perform the convergence analysis later on.

3.1. A uniform $L^{\infty}$-estimate on $s_{\mathcal{M}, \Delta t}$. In what follows, $\left(p_{K}^{n+1}\right)_{K \in \mathcal{V}, n \geq 0}$ denotes a solution to the scheme (20) (whose existence will be etablished later). This allows to define the quantities $s_{K}^{n+1}=s\left(p_{K}^{n+1}\right)$ and $\xi_{K}^{n+1}=\xi\left(p_{K}^{n+1}\right)$ for all $K \in \mathcal{V}$ and all $n \in\{0, \ldots, N\}$.

Proposition 3.1. For all $K \in \mathcal{V}$, and all $n \in\{0, \ldots, N\}$, one has

$$
0 \leq s_{K}^{n} \leq 1
$$

Equivalently, one has

$$
p_{\star} \leq p_{K}^{n+1}, \quad \forall K \in \mathcal{V}, \forall n \in\{0, \ldots, N\} .
$$

Proof. First of all, note that there is nothing to prove if $p_{\star}=-\infty$. Therefore, we restrict our attention to the case of a finite $p_{\star}$. The property (28) holds for $n=0$ thanks to the discretization (19) of the initial data. Assume now (28) holds at time step $n$. It is equivalent to prove $p_{K}^{n+1} \geq p_{\star}$. Assume that

$$
p_{K_{m}}^{n+1}=\min _{L \in \mathcal{V}} p_{L}^{n+1}<p_{\star} \quad \Leftrightarrow \quad s_{K_{m}}^{n+1}<0 .
$$

In view of the definition $(21)$ of $\eta_{K_{m} L}^{n+1}$, and of the fact that $\eta(s)=0$ if $s<0$, it follows from (20d) that

$$
\eta_{K_{m} L}^{n+1}=0 \quad \text { if } \quad a_{K_{m} L}\left(u_{K_{m}}^{n+1}-u_{L}^{n+1}\right) \geq 0 .
$$

Therefore, the scheme (20) at vertex $K_{m}$ rewrites

$$
s_{K_{m}}^{n+1}=s_{K_{m}}^{n}-\frac{\Delta t}{m_{K_{m}}} \sum_{\sigma_{K_{m} L} \in \mathcal{E}} \eta_{K_{m} L}^{n+1} a_{K_{m} L}\left(u_{K_{m}}^{n+1}-u_{L}^{n+1}\right) \geq 0 .
$$

This yields a contradiction with (30). Hence, the $L^{\infty}$ estimate (28) holds at the time step $n+1$, thus for all $n$.

3.2. Capillary energy estimate and the control of the dissipation. The goal of this section is to get an a priori control for the capillary energy of the discrete solution and to derive some estimates coming from the dissipation of the energy. We were not able to derive the discrete counterpart of the energy/dissipation estimate (10). However, we can prove a discrete counterpart of (11) (cf. Proposition 3.2) that appears to be sufficient to establish Theorems 2.4 and 2.5. In what follows, we assume that $\left(s_{K}^{n}\right)_{K \in \mathcal{V}}$ is known and $\left(p_{K}^{n+1}\right)_{K \in \mathcal{V}}$ denotes an arbitrary solution to the scheme (20).

Proposition 3.2. There exists $C_{4}$ depending only on $\theta_{\mathcal{T}}, \Lambda, \Omega, t_{f},\|s\|_{L^{1}\left(p_{\star}, 0\right)}$, and $\|\eta\|_{\infty}$ such that

$$
\sup _{n \in\{0, \ldots, N\}} \sum_{K \in \mathcal{V}} m_{K} \Gamma\left(p_{K}^{n+1}\right)+\sum_{n=0}^{N} \Delta t \sum_{\sigma_{K L} \in \mathcal{E}} a_{K L}\left(\xi\left(p_{K}^{n+1}\right)-\xi\left(p_{L}^{n+1}\right)\right)^{2} \leq C_{4} .
$$


The proof of Proposition 3.2 is based on several Lemmas stated below. This section also contains technical lemmas that will be useful in the convergence proof of $\S 4$.

Lemma 3.3. There exists $C_{5}$ depending only on $\Omega, s$ such that, for all $\nu \in\{0, \ldots, N\}$, one has

(31) $\sum_{K \in \mathcal{V}} m_{K} \Gamma\left(p_{K}^{\nu+1}\right)+\sum_{n=0}^{\nu} \Delta t \sum_{\sigma_{K L} \in \mathcal{E}} a_{K L} \eta_{K L}^{n+1}\left(u_{K}^{n+1}-u_{L}^{n+1}\right)\left(p_{K}^{n+1}-p_{L}^{n+1}\right) \leq C_{5}$.

Proof. We multiply the scheme (20a) by $\Delta t p_{K}^{n+1}$ and sum on $K \in \mathcal{V}$. This yields:

$$
A+B=0,
$$

where

$$
A=\sum_{K \in \mathcal{V}} m_{K}\left(s_{K}^{n+1}-s_{K}^{n}\right) p_{K}^{n+1}, \quad B=\Delta t \sum_{K \in \mathcal{V}} \sum_{\sigma_{K L} \in \mathcal{E}} a_{K L} \eta_{K L}^{n+1}\left(u_{K}^{n+1}-u_{L}^{n+1}\right) p_{K}^{n+1} .
$$

Since $a_{K L}=a_{L K}$ and $\eta_{K L}^{n+1}=\eta_{L K}^{n+1}$, we can rewrite

$$
B=\Delta t \sum_{\sigma_{K L} \in \mathcal{E}} a_{K L} \eta_{K L}^{n+1}\left(u_{K}^{n+1}-u_{L}^{n+1}\right)\left(p_{K}^{n+1}-p_{L}^{n+1}\right)
$$

By convexity of $\Gamma \circ s^{-1}$ one deduces this estimation

$$
A \geq \sum_{K \in V} m_{K}\left(\Gamma\left(p_{K}^{n+1}\right)-\Gamma \circ s^{-1}\left(s_{K}^{n}\right)\right) .
$$

Summing over $n \in\{0, \ldots, \nu\}$ provides

$$
\begin{array}{r}
\sum_{K \in \mathcal{V}} m_{K} \Gamma\left(p_{K}^{\nu+1}\right)+\sum_{n=0}^{\nu} \Delta t \sum_{\sigma_{K L} \in \mathcal{E}} a_{K L} \eta_{K L}^{n+1}\left(u_{K}^{n+1}-u_{L}^{n+1}\right)\left(p_{K}^{n+1}-p_{L}^{n+1}\right) \\
\leq \sum_{K \in \mathcal{V}} m_{K} \Gamma \circ s^{-1}\left(s_{K}^{0}\right)
\end{array}
$$

It remains to check that for $b \in[0,1]$

$$
0 \leq \Gamma \circ s^{-1}(b)=\int_{0}^{s^{-1}(b)} a s^{\prime}(a) \mathrm{d} a=\int_{1}^{b} s^{-1}(a) \mathrm{d} a \leq\left\|s^{-1}\right\|_{L^{1}(0,1)}<+\infty
$$

ensuring that

$$
\sum_{K \in \mathcal{V}} m_{K} \Gamma \circ s^{-1}\left(s_{K}^{0}\right) \leq \int_{\Omega} \Gamma \circ s^{-1}\left(s_{0}\right) \mathrm{d} \boldsymbol{x} \leq|\Omega|\left\|s^{-1}\right\|_{L^{1}(0,1)}
$$

thanks to Jensen's inequality and to (5).

From the previous lemma, we can get an estimate on the spatial variations of the function $\xi_{\mathcal{T}, \Delta t}$. In order to ease the reading, we use the shortened notation

$$
\xi_{K}^{n+1}=\xi\left(p_{K}^{n+1}\right), \quad \forall K \in \mathcal{V}, \forall n \in\{0, \ldots, N\}
$$

Lemma 3.4. There exists $C_{6}$ depending only on $\Omega, s, t_{f}, \Lambda, \theta_{\mathcal{T}}$, and $\eta$ such that

$$
\iint_{Q_{t_{f}}} \Lambda \nabla \xi_{\mathcal{T}, \Delta t} \cdot \nabla \xi_{\mathcal{T}, \Delta t} \mathrm{~d} \boldsymbol{x} \mathrm{d} t=\sum_{n=0}^{N} \Delta t \sum_{\sigma_{K L} \in \mathcal{E}} a_{K L}\left(\xi_{K}^{n+1}-\xi_{L}^{n+1}\right)^{2} \leq C_{6}
$$


Proof. The definition (21) of the mobilities $\eta_{K L}^{n+1}$ has been chosen so that

$$
\begin{aligned}
C_{5} \geq \sum_{n=0}^{N} \Delta t \sum_{\sigma_{K L} \in \mathcal{E}} a_{K L} \eta_{K L}^{n+1}\left(u_{K}^{n+1}-u_{L}^{n+1}\right)\left(p_{K}^{n+1}-p_{L}^{n+1}\right) \\
\geq \sum_{n=0}^{N} \Delta t \sum_{\sigma_{K L} \in \mathcal{E}} a_{K L} \tilde{\eta}_{K L}^{n+1}\left(u_{K}^{n+1}-u_{L}^{n+1}\right)\left(p_{K}^{n+1}-p_{L}^{n+1}\right),
\end{aligned}
$$

where $\tilde{\eta}_{K L}^{n+1}=\eta\left(s\left(p_{K L}\right)\right)$ whatever $p_{K L} \in I_{K L}^{n+1}$. Therefore, using the definition (20b) of $u_{K}^{n+1}$ and Young's inequality leads to

$$
\begin{aligned}
C_{5} \geq & \sum_{n=0}^{N} \Delta t \sum_{\sigma_{K L} \in \mathcal{E}} a_{K L} \tilde{\eta}_{K L}^{n+1}\left(\left(p_{K}^{n+1}-p_{L}^{n+1}\right)^{2}+\left(p_{K}^{n+1}-p_{L}^{n+1}\right)\left(z_{K}-z_{L}\right)\right) \\
\geq & \sum_{n=0}^{N} \Delta t \sum_{\sigma_{K L} \in \mathcal{E}} a_{K L} \tilde{\eta}_{K L}^{n+1}\left(p_{K}^{n+1}-p_{L}^{n+1}\right)^{2} \\
& -\frac{\alpha}{2} \sum_{n=0}^{N} \Delta t \sum_{\sigma_{K L} \in \mathcal{E}}\left|a_{K L}\right| \tilde{\eta}_{K L}^{n+1}\left(p_{K}^{n+1}-p_{L}^{n+1}\right)^{2}-\frac{\|\eta\|_{\infty}}{2 \alpha} \sum_{n=0}^{N} \Delta t \sum_{\sigma_{K L} \in \mathcal{E}}\left|a_{K L}\right|\left(z_{K}-z_{L}\right)^{2}
\end{aligned}
$$

where $\alpha$ is a positive parameter to be fixed. We choose

$$
\tilde{\eta}_{K L}^{n+1}=\left\{\begin{array}{lll}
\left(\frac{\xi_{K}^{n+1}-\xi_{L}^{n+1}}{p_{K}^{n+1}-p_{L}^{n+1}}\right)^{2} & \text { if } & p_{K}^{n+1} \neq p_{L}^{n+1}, \\
\eta\left(s_{K}^{n+1}\right) & \text { if } & p_{K}^{n+1}=p_{L}^{n+1}
\end{array}\right.
$$

leading to

$$
\begin{aligned}
\sum_{n=0}^{N} \Delta t \sum_{\sigma_{K L} \in \mathcal{E}} a_{K L}\left(\xi_{K}^{n+1}-\xi_{L}^{n+1}\right)^{2} & -\frac{\alpha}{2} \sum_{n=0}^{N} \Delta t \sum_{\sigma_{K L} \in \mathcal{E}}\left|a_{K L}\right|\left(\xi_{K}^{n+1}-\xi_{L}^{n+1}\right)^{2} \\
& \leq C_{5}+\frac{\|\eta\|_{\infty}}{2 \alpha} \sum_{n=0}^{N} \Delta t \sum_{\sigma_{K L} \in \mathcal{E}}\left|a_{K L}\right|\left(z_{K}-z_{L}\right)^{2} .
\end{aligned}
$$

Using Lemma 2.1, we get that

$$
\left(1-\frac{\alpha C_{3}}{2}\right) \sum_{n=0}^{N} \Delta t \sum_{\sigma_{K L} \in \mathcal{E}} a_{K L}\left(\xi_{K}^{n+1}-\xi_{L}^{n+1}\right)^{2} \leq C_{5}+\frac{\|\eta\|_{\infty}}{2 \alpha} t_{f} C_{3}|\Omega| .
$$

We conclude the proof by setting $\alpha=\frac{1}{C_{3}}$.

The function $\Gamma$ takes non-negative values, hence so does the first term in (31). But since $a_{K L}$ may become negative, we are not able to claim that the second term is non-negative (this would end the proof of Proposition 3.2). Nevertheless, we can prove that this term is uniformly bounded. This information, combined with Lemma 3.4, is sufficient to conclude the proof of Proposition 3.2.

Lemma 3.5. There exists $C_{7}$ depending only on $\Omega, s, t_{f}, \Lambda, \theta_{\mathcal{T}}$, and $\eta$ such that

$$
\sum_{n=0}^{N} \Delta t \sum_{\sigma_{K L} \in \mathcal{E}}\left|a_{K L}\right| \eta_{K L}^{n+1}\left|u_{K}^{n+1}-u_{L}^{n+1}\right|\left|p_{K}^{n+1}-p_{L}^{n+1}\right| \leq C_{7} .
$$


Proof. Since $|x|=x+2 x^{-}, x^{-}=\max (-x, 0)$, one has

$$
\begin{aligned}
\sum_{n=0}^{N} \Delta t & \sum_{\sigma_{K L} \in \mathcal{E}}\left|a_{K L}\right| \eta_{K L}^{n+1}\left|u_{K}^{n+1}-u_{L}^{n+1}\right|\left|p_{K}^{n+1}-p_{L}^{n+1}\right| \\
=\sum_{n=0}^{N} \Delta t & \sum_{\sigma_{K L} \in \mathcal{E}} a_{K L} \eta_{K L}^{n+1}\left(u_{K}^{n+1}-u_{L}^{n+1}\right)\left(p_{K}^{n+1}-p_{L}^{n+1}\right) \\
& \quad+2 \sum_{n=0}^{N} \Delta t \sum_{\sigma_{K L} \in \mathcal{E}} \eta_{K L}^{n+1}\left[a_{K L}\left(u_{K}^{n+1}-u_{L}^{n+1}\right)\left(p_{K}^{n+1}-p_{L}^{n+1}\right)\right]^{-} .
\end{aligned}
$$

It follows from the definition (21) of $\eta_{K L}^{n+1}$ that

$\eta_{K L}^{n+1}\left[a_{K L}\left(u_{K}^{n+1}-u_{L}^{n+1}\right)\left(p_{K}^{n+1}-p_{L}^{n+1}\right)\right]^{-} \leq \tilde{\eta}_{K L}^{n+1}\left[a_{K L}\left(u_{K}^{n+1}-u_{L}^{n+1}\right)\left(p_{K}^{n+1}-p_{L}^{n+1}\right)\right]^{-}$, with $\tilde{\eta}_{K L}^{n+1}$ defined by (34). Moreover, using the definition (20b) of $u_{K}^{n+1}$ together with Young's inequality, we obtain that

$$
\begin{aligned}
& \tilde{\eta}_{K L}^{n+1}\left[a_{K L}\left(u_{K}^{n+1}-u_{L}^{n+1}\right)\left(p_{K}^{n+1}-p_{L}^{n+1}\right)\right]^{-} \\
& \leq \tilde{\eta}_{K L}^{n+1}\left|a_{K L}\right|\left(p_{K}^{n+1}-p_{L}^{n+1}\right)^{2}+\tilde{\eta}_{K L}^{n+1}\left|a_{K L}\right|\left|z_{K}-z_{L}\right|\left|p_{K}^{n+1}-p_{L}^{n+1}\right| \\
& \leq\left|a_{K L}\right|\left(\xi_{K}^{n+1}-\xi_{L}^{n+1}\right)^{2}+\frac{1}{2}\left|a_{K L}\right| \tilde{\eta}_{K L}^{n+1}\left(p_{K}^{n+1}-p_{L}^{n+1}\right)^{2}+\frac{1}{2}\left|a_{K L}\right| \tilde{\eta}_{K L}^{n+1}\left(z_{K}-z_{L}\right)^{2} \\
& \leq \frac{3}{2}\left|a_{K L}\right|\left(\xi_{K}^{n+1}-\xi_{L}^{n+1}\right)^{2}+\frac{\|\eta\|_{\infty}}{2}\left|a_{K L}\right|\left(z_{K}-z_{L}\right)^{2} .
\end{aligned}
$$

We deduce from Lemma 2.1 that

$$
\begin{aligned}
2 \sum_{n=0}^{N} \Delta t \sum_{\sigma_{K L} \in \mathcal{E}} \eta_{K L}^{n+1}\left[a_{K L}\left(u_{K}^{n+1}-u_{L}^{n+1}\right)\left(p_{K}^{n+1}-p_{L}^{n+1}\right)\right]^{-} \\
\quad \leq 3 C_{3} \sum_{n=0}^{N} \Delta t \sum_{\sigma_{K L} \in \mathcal{E}} a_{K L}\left(\xi_{K}^{n+1}-\xi_{L}^{n+1}\right)^{2}+C_{3}\|\eta\|_{\infty} t_{f}|\Omega|
\end{aligned}
$$

Then we combine (35), (36), Lemma 3.4, and Lemma 3.3 to conclude.

The a priori estimate of Proposition 3.2 follows easily from Lemmas 3.3, 3.4, and 3.5. It is sufficient to prove the existence of a solution to the scheme (20) (see §3.3). Nevertheless, before going to this existence proof, we still provide some additional a priori estimates to be used later on in $\S 4$.

Lemma 3.6. There exists $C_{8}$ depending only on $\Omega, s, t_{f}, \Lambda, \theta_{\mathcal{T}}$, and $\eta$ such that

$$
\begin{aligned}
& \sum_{n=0}^{N} \Delta t \sum_{\sigma_{K L} \in \mathcal{E}}\left|a_{K L}\right| \eta_{K L}^{n+1}\left(p_{K}^{n+1}-p_{L}^{n+1}\right)^{2} \leq C_{8}, \\
& \sum_{n=0}^{N} \Delta t \sum_{\sigma_{K L} \in \mathcal{E}}\left|a_{K L}\right| \eta_{K L}^{n+1}\left(u_{K}^{n+1}-u_{L}^{n+1}\right)^{2} \leq C_{8} .
\end{aligned}
$$

Proof. The definition (20b) of $u_{K}^{n+1}$ yields

$$
\sum_{n=0}^{N} \Delta t \sum_{\sigma \in \mathcal{E}_{K L}}\left|a_{K L}\right| \eta_{K L}^{n+1}\left(p_{K}^{n+1}-p_{L}^{n+1}\right)^{2}=A+B,
$$


where

$$
\begin{aligned}
& A=\sum_{n=0}^{N} \Delta t \sum_{\sigma \in \mathcal{E}_{K L}}\left|a_{K L}\right| \eta_{K L}^{n+1}\left(p_{K}^{n+1}-p_{L}^{n+1}\right)\left(u_{K}^{n+1}-u_{L}^{n+1}\right), \\
& B=-\sum_{n=0}^{N} \Delta t \sum_{\sigma \in \mathcal{E}_{K L}}\left|a_{K L}\right| \eta_{K L}^{n+1}\left(p_{K}^{n+1}-p_{L}^{n+1}\right)\left(z_{K}^{n+1}-z_{L}^{n+1}\right) .
\end{aligned}
$$

Thanks to Lemma 3.5, one has $A \leq C_{7}$. Moreover, combining once again Young inequality with Lemma 2.1, we get that

$$
B \leq \frac{1}{2} \sum_{n=0}^{N} \Delta t \sum_{\sigma \in \mathcal{E}_{K L}}\left|a_{K L}\right| \eta_{K L}^{n+1}\left(p_{K}^{n+1}-p_{L}^{n+1}\right)^{2}+C_{3}\|\eta\|_{\infty} t_{f}|\Omega|
$$

hence (37) holds with $C_{8}=2 C_{7}+2 C_{3}\|\eta\|_{\infty} t_{f}|\Omega|$. The proof of (38) is similar.

The last lemma of this section is devoted to the control of the $L^{2}$ norm of $\xi_{\mathcal{T}, \Delta t}$. Lemma 3.4 only provides a control on the gradient of $\xi_{\mathcal{T}, \Delta t}$, but not on $\xi_{\mathcal{T}, \Delta t}$ directly. The control on $\xi_{\mathcal{T}, \Delta t}$ is provided by an argument à la Poincaré, cf. Appendix A.1.

Lemma 3.7. There exists $C_{9}$ depending only on $\Omega, t_{f}, s, \Lambda, \theta_{\mathcal{T}}, \eta, \bar{s}_{0}$, and $\xi_{\star}$ such that

$$
\begin{aligned}
& \left\|\xi_{\mathcal{T}, \Delta t}\right\|_{L^{2}\left(Q_{t_{f}}\right)} \mathrm{d} \boldsymbol{x} \mathrm{d} t \leq C_{9} \\
& \left\|\xi_{\mathcal{M}, \Delta t}\right\|_{L^{2}\left(Q_{t_{f}}\right)} \mathrm{d} \boldsymbol{x} \mathrm{d} t \leq C_{9}
\end{aligned}
$$

Proof. Let us first etablish (40). Thanks to Assumption (4), we know that $\int_{\Omega} s_{0} \mathrm{~d} \boldsymbol{x}<$ meas $(\Omega)$. The global conservativity property (23) allows to claim that

$$
\sum_{K \in \mathcal{V}} s_{K}^{n+1} m_{K}=\bar{s}_{0}=\int_{\Omega} s_{0}(\boldsymbol{x}) \mathrm{d} \boldsymbol{x}<\operatorname{meas}(\Omega)
$$

for any $n \in\{0, \ldots, N\}$. Using that $\xi_{K}^{n+1}<0$ if and only if $s_{K}^{n+1}<1$ (recall that $\xi(p)<0$ iff $p<0$ iff $s<1$ ), one gets

$$
\operatorname{meas}\left\{\xi_{\mathcal{M}, \Delta t}\left(\cdot, t_{n+1}\right)<0\right\} \geq \operatorname{meas}(\Omega)-\bar{s}_{0}>0 \text {. }
$$

Denote by $\xi_{K}^{+, n+1}=\max \left(0, \xi_{K}^{n+1}\right)$, and by $\xi_{\mathcal{M}, \Delta t}^{+}$and $\xi_{\mathcal{T}, \Delta t}^{+}$the unique elements of $X_{\mathcal{M}, \Delta t}$ and $V_{\mathcal{T}, \Delta t}$ respectively such that

$$
\xi_{\mathcal{M}, \Delta t}^{+}\left(\boldsymbol{x}_{K}, t_{n+1}\right)=\xi_{\mathcal{T}, \Delta t}^{+}\left(\boldsymbol{x}_{K}, t_{n+1}\right)=\xi_{K}^{+, n+1}, \quad \forall K \in \mathcal{V}, \forall n \in\{0, \ldots, N\}
$$


Note that $\xi_{\mathcal{T}, \Delta t}^{+} \neq\left(\xi_{\mathcal{T}, \Delta t}\right)^{+}=\max \left(0, \xi_{\mathcal{T}, \Delta t}\right)$ in general, but that $\xi_{\mathcal{M}, \Delta t}^{+}=\left(\xi_{\mathcal{M}, \Delta t}\right)^{+}$ and that $\xi_{\mathcal{M}, \Delta t}^{-}=\left(\xi_{\mathcal{M}, \Delta t}\right)^{-}=\max \left(0,-\xi_{\mathcal{M}, \Delta t}\right)$. Using Assumption (A3), the 1Lipschitz continuity of $x \mapsto x^{+}$, and Lemmas 2.1 and 3.4, we obtain

$$
\begin{aligned}
\iint_{Q_{t_{f}}}\left|\nabla \xi_{\mathcal{T}, \Delta t}^{+}\right|^{2} \mathrm{~d} \boldsymbol{x} \mathrm{d} t \leq \frac{1}{\underline{\Lambda}} \sum_{n=0}^{N} \Delta t \sum_{\sigma_{K L} \in \mathcal{E}} a_{K L}\left(\xi_{K}^{+, n+1}-\xi_{L}^{+, n+1}\right)^{2} \\
\leq \frac{1}{\underline{\Lambda}} \sum_{n=0}^{N} \Delta t \sum_{\sigma_{K L} \in \mathcal{E}}\left|a_{K L}\right|\left(\xi_{K}^{+, n+1}-\xi_{L}^{+, n+1}\right)^{2} \\
\quad \leq \frac{1}{\underline{\Lambda}} \sum_{n=0}^{N} \Delta t \sum_{\sigma_{K L} \in \mathcal{E}}\left|a_{K L}\right|\left(\xi_{K}^{n+1}-\xi_{L}^{n+1}\right)^{2} \leq \frac{C_{3} C_{6}}{\underline{\Lambda}} .
\end{aligned}
$$

Therefore, we can apply Lemma A.3 stated in appendix. This provides

$$
\iint_{Q_{t_{f}}}\left(\xi_{\mathcal{M}, \Delta t}^{+}\right)^{2} \mathrm{~d} \boldsymbol{x} \mathrm{d} t \leq C
$$

On the other hand, because of (3), we know that $\xi_{\mathcal{M}, \Delta t}^{-} \leq \xi_{\star}$ a.e. in $Q_{t_{f}}$, hence

$$
\iint_{Q_{t_{f}}}\left(\xi_{\mathcal{M}, \Delta t}^{-}\right)^{2} \mathrm{~d} \boldsymbol{x} \mathrm{d} t \leq\left(\xi_{\star}\right)^{2} \operatorname{meas}(\Omega) t_{f}
$$

Combining (42) with (43) provides (40). In order to recover (39), in only remains to use (13) and (40).

3.3. Existence of a discrete solution. In order to prove the existence of a solution $\left(p_{K}^{n+1}\right)_{K}$ to the scheme (20), we need an additional mesh-depending estimate on the solution. Following [9], we introduce now the notion of transmissive path.

Definition 3.8. A transmissive path $w$ joining $K_{i} \in \mathcal{V}$ to $K_{f} \in \mathcal{V}$ consists in a list of vertices $\left(K_{q}\right)_{0 \leq q \leq M}$ such that $K_{i}=K_{0}, K_{f}=K_{M}$, with $K_{q} \neq K_{\ell}$ if $q \neq \ell$, and such that $\sigma_{K_{q} K_{q+1}} \in \mathcal{E}$ with $a_{K_{q} K_{q+1}}>0$ for all $q \in\{0, \ldots, M-1\}$. We denote by $\mathcal{W}\left(K_{i}, K_{f}\right)$ the set of the transmissive path joining $K_{i} \in \mathcal{V}$ to $K_{f} \in \mathcal{V}$.

We now state a result which is proved in [9, Lemma 3.5].

Lemma 3.9. For all $\left(K_{i}, K_{f}\right) \in \mathcal{V}^{2}$ there exists a transmissive path $w \in \mathcal{W}\left(K_{i}, K_{f}\right)$.

Lemma 3.10. There exists $C_{\star}>-\infty$ depending only on $\mathcal{T}, \Delta t, \Omega, s, \bar{s}_{0}, t_{f}, \Lambda$, $\theta_{\mathcal{T}}, \eta$ and $z$ such that

$$
p_{K}^{n+1} \geq C_{\star}, \quad \forall K \in \mathcal{V}, \quad \forall n \in\{0, \ldots, N\} .
$$

Proof. Let us prove that $p_{K}^{n+1} \geq C_{\star}$. Assume first that $p_{\star}>-\infty$, then we can choose $C_{\star}=p_{\star}$ thanks to (29), so that we can focus on the case $p_{\star}=-\infty$.

In view of the global conservation property (23), one has that

$$
\sum_{K \in \mathcal{V}}\left(s_{K}^{n+1}-\bar{s}_{0}\right) m_{K}=0
$$


This ensures the existence of at least one vertex $K_{i}$ such that $s_{K_{i}}^{n+1} \geq \bar{s}_{0}>0$. In particular,

$$
-\infty<s^{-1}\left(\bar{s}_{0}\right) \leq p_{K_{i}}^{n+1}
$$

Let $K_{f} \in \mathcal{V} \backslash\left\{K_{i}\right\}$, then thanks to Lemma 3.9, there exists a transmissive path $w \in \mathcal{W}\left(K_{i}, K_{f}\right)=\left(K_{q}\right)_{0 \leq q \leq M}$ of finite length in the sense of Definition 3.8. Let us show that for all $p_{K_{q}}^{n+1}>-\infty$ for all $q \in\{0, \ldots, M\}$.

First, we have checked in (44) that $p_{K_{0}}^{n+1}>-\infty$. Assume now that $p_{K_{q}}^{n+1}>-\infty$ for some $q \in\{0, \ldots, M-1\}$, then it follows from Lemma 3.5 that

$$
\sum_{n=0}^{N} \Delta t \sum_{\sigma_{K L} \in \mathcal{E}}\left|a_{K L}\right| \eta_{K L}^{n+1}\left|u_{K}^{n+1}-u_{L}^{n+1}\right|\left|p_{K}^{n+1}-p_{L}^{n+1}\right| \leq C_{6} .
$$

This ensures in particular that

$$
\Delta t a_{K_{q} K_{q+1}} \eta_{K_{q} K_{q+1}}^{n+1}\left(u_{K_{q}}^{n+1}-u_{K_{q+1}}^{n+1}\right)\left(p_{K_{q}}^{n+1}-p_{K_{q+1}}^{n+1}\right) \leq C_{6}
$$

Thanks to the definition (21) of $\eta_{K_{q} K_{q+1}}^{n+1}$, one has

$$
\begin{aligned}
& a_{K_{q} K_{q+1}} \eta_{K_{q} K_{q+1}}^{n+1}\left(u_{K_{q}}^{n+1}-u_{K_{q+1}}^{n+1}\right)\left(p_{K_{q}}^{n+1}-p_{K_{q+1}}^{n+1}\right) \\
& \quad \geq a_{K_{q} K_{q+1}} \eta\left(s_{K_{q}}^{n+1}\right)\left(u_{K_{q}}^{n+1}-u_{K_{q+1}}^{n+1}\right)\left(p_{K_{q}}^{n+1}-p_{K_{q+1}}^{n+1}\right) .
\end{aligned}
$$

Since $a_{K_{q} K_{q+1}}>0$, we obtain that

$$
\begin{aligned}
& \left(u_{K_{q}}^{n+1}-u_{K_{q+1}}^{n+1}\right)\left(p_{K_{q}}^{n+1}-p_{K_{q+1}}^{n+1}\right) \\
& =\left(p_{K_{q}}^{n+1}-p_{K_{q+1}}^{n+1}\right)^{2}+\left(p_{K_{q}}^{n+1}-p_{K_{q+1}}^{n+1}\right)\left(z_{K_{q}}-z_{K_{q+1}}\right) \leq \frac{C_{6}}{\Delta t a_{K_{q} K_{q+1}} \eta\left(s\left(p_{K_{q}}^{n+1}\right)\right)} .
\end{aligned}
$$

Using Young inequality one has

$$
\left(u_{K_{q}}^{n+1}-u_{K_{q+1}}^{n+1}\right)\left(p_{K_{q}}^{n+1}-p_{K_{q+1}}^{n+1}\right) \geq \frac{1}{2}\left(p_{K_{q}}^{n+1}-p_{K_{q+1}}^{n+1}\right)^{2}-\frac{1}{2}\left(z_{K_{q}}-z_{K_{q+1}}\right)^{2},
$$

thus

$$
p_{K_{q+1}}^{n+1} \geq p_{K_{q}}^{n+1}-\sqrt{\left(z_{K_{q}}-z_{K_{q+1}}\right)^{2}+\frac{2 C_{6}}{\Delta t a_{K_{q} K_{q+1}} \eta\left(s\left(p_{K_{q}}^{n+1}\right)\right)}}
$$

This ensures that $p_{K_{q+1}}^{n+1}>-\infty$.

We have proved the existence of a finite quantity $\left(C_{K_{i}, K_{f}, w}\right)_{K_{f} \in \mathcal{V}}$ (depending on the data of the continuous problem $\Omega, s, \bar{s}_{0}, t_{f}, \Lambda, \theta_{\mathcal{T}}, \eta$ but also on the discretization $\mathcal{T}$ and on $\Delta t$ ) such that

$$
s\left(p_{K_{i}}^{n+1}\right) \geq \bar{s}_{0} \quad \Longrightarrow \quad p_{K_{f}}^{n+1} \geq-C_{K_{i}, K_{f}, w}
$$

As a consequence, since there exists a finite number of transmissive paths between two vertices, we get the estimate

$$
p_{K}^{n+1} \geq-\max _{K_{i} \in \mathcal{V}} \max _{K_{f} \in \mathcal{V}} \min _{w \in \mathcal{W}\left(K_{i}, K_{f}\right)} C_{K_{i}, K_{f}, w}>-\infty, \quad \forall K \in \mathcal{V}, \forall n \in\{0, \ldots, N\}
$$

In the previous lemma, we managed to bound the $\left\{p_{K}^{n+1}\right\}$ from below. The next lemma provides a bound from above. 
Lemma 3.11. There exists $p^{\star}<\infty$ depending only on $\mathcal{T}, \Delta t, \Omega, t_{f}, s, \Lambda, \eta, \bar{s}_{0}$ and $\xi_{\star}$ such that

$$
p_{K}^{n+1} \leq p^{\star} \quad \forall K \in \mathcal{V}, \quad \forall n \in\{0, \ldots, N\} .
$$

Proof. Since $s(p)=1$ if $p \geq 0$, one has $\xi(p)=p \sqrt{\eta(1)}$ if $p \geq 0$. By (40), one has

$$
\Delta t m_{K} \xi\left(p_{K}^{n+1}\right)^{2} \leq\left\|\xi_{\mathcal{M}, \Delta t}\right\|_{L^{2}\left(Q_{t_{f}}\right)}^{2} \leq\left(C_{9}\right)^{2} .
$$

Therefore, we get $p_{K}^{n+1} \leq \frac{C_{9}}{\sqrt{\Delta t m_{K}}} \frac{1}{\eta(1)}$.

Now, one can apply the same strategy as in [9, Lemma 3.11] for proving the existence of a solution to the scheme (20).

Proposition 3.12. Let $\left(s_{K}^{n}\right)_{K \in \mathcal{V}} \in[0,1]^{\# \mathcal{V}}$ be such that $\sum_{K \in \mathcal{V}} m_{K} s_{K}^{n}=\operatorname{meas}(\Omega) \bar{s}_{0}$, there exists (at least) one solution $\left(p_{K}^{n+1}\right)_{K \in \mathcal{V}} \in\left[p_{\star}, p^{\star}\right]^{\# \mathcal{V}}$ of the scheme (20). Moreover, it satisfies $\sum_{K \in \mathcal{V}} m_{K} s_{K}^{n+1}=\operatorname{meas}(\Omega) \bar{s}_{0}$.

The proof of Proposition 3.12 is not detailed here since it mimics the one of [9, Lemma 3.11]. Let us just mention that it is based on a topological degree argument $[25,12]$.

\section{Convergence towards a weak solution}

The proof of the convergence properties stated in Theorem 2.5 is based on compactness arguments. As a first step, we show in $\S 4.1$ the appropriate compactness properties on the reconstructed discrete solutions. Then we identify in $\S 4.2$ the limit value (whose existence is ensured thanks to the compactness properties) as the unique weak solution to the problem (1).

4.1. Compactness properties of discrete solutions. As it is classical for unsteady problems, we need to prove some time-compactness for the approximate solutions. Because of the degeneracy of the problem we consider, we cannot use a strategy à la Aubin-Simon [29] (see [21] for an extension of this strategy to the discrete setting). A classical way to circumvent this problem is to estimate the time-translates (see [3] in the continuous setting and [15] in the discrete setting). This strategy could have been used here, but we rather make use of the timecompactness result for degenerate parabolic equations proposed in [4]. To this end, we need the following lemma.

Lemma 4.1. There exists $C_{10}$ depending only on $\Omega, s, t_{f}, \Lambda, \theta_{\mathcal{T}}, z$ and $\eta$ such that

$$
\sum_{n=0}^{N} \sum_{K \in \mathcal{V}}\left(s_{K}^{n+1}-s_{K}^{n}\right) \psi\left(\boldsymbol{x}_{K}, t_{n+1}\right) m_{K} \leq C_{10}\|\nabla \psi\|_{\infty}, \quad \forall \psi \in \mathcal{C}_{c}^{\infty}\left(Q_{t_{f}}\right) .
$$

Proof. For the sake of readability, we denote by $\psi_{K}^{n+1}=\psi\left(\boldsymbol{x}_{K}, t_{n+1}\right)$ for all $K \in \mathcal{V}$ and all $n \in\{0, \ldots, M\}$. We multiply (20a) by $\Delta t \psi_{K}^{n+1}$ and sum for $K \in \mathcal{V}$, for $n \in\{0, \ldots, N\}$. This yields

$$
A=B
$$


where

$$
\begin{aligned}
A & =\sum_{n=0}^{N} \sum_{K \in \mathcal{V}} m_{K}\left(s_{K}^{n+1}-s_{K}^{n}\right) \psi_{K}^{n+1}, \\
B & =-\sum_{n=0}^{N} \Delta t \sum_{\sigma_{K L} \in \mathcal{E}} a_{K L} \eta_{K L}^{n+1}\left(u_{K}^{n+1}-u_{L}^{n+1}\right)\left(\psi_{K}^{n+1}-\psi_{L}^{n+1}\right) .
\end{aligned}
$$

Using the Cauchy-Schwarz inequality, we get

$$
\begin{aligned}
|B|^{2} \leq\left(\sum_{n=0}^{N} \Delta t \sum_{\sigma_{K L} \in \mathcal{E}}\left|a_{K L}\right| \eta_{K L}^{n+1}\left(u_{K}^{n+1}-u_{L}^{n+1}\right)^{2}\right) \\
\times\left(\sum_{n=0}^{N} \Delta t \sum_{\sigma_{K L} \in \mathcal{E}}\left|a_{K L}\right| \eta_{K L}^{n+1}\left(\psi_{K}^{n+1}-\psi_{L}^{n+1}\right)^{2}\right) .
\end{aligned}
$$

Using Lemma 3.6, the boundedness of $\eta$ and Lemma 2.1, we obtain that

$$
|B|^{2} \leq\|\eta\|_{\infty} C_{8} C_{3} \iint_{Q_{t_{f}}} \Lambda \nabla \psi_{\mathcal{T}, \Delta t} \cdot \nabla \psi_{\mathcal{T}, \Delta t} \leq\|\eta\|_{\infty} C_{8} C_{3} \operatorname{meas}(\Omega) t_{f} \bar{\Lambda}\|\nabla \psi\|_{\infty}^{2}
$$

Therefore (45) holds with $C_{10}=\sqrt{\|\eta\|_{\infty} C_{8} C_{3} \operatorname{meas}(\Omega) t_{f} \bar{\Lambda}}$

We can now state the expected compactness properties.

Proposition 4.2. There exists a measurable function $p: Q_{t_{f}} \longrightarrow\left[p_{\star}, p^{\star}\right]$ such that, up to an unlabeled subsequence, one has

$$
\begin{aligned}
& s_{\mathcal{M}_{m}, \Delta t_{m}} \underset{m \rightarrow+\infty}{\longrightarrow} s(p) \quad \text { a.e in } Q_{t_{f}}, \\
& \xi_{\mathcal{T}_{m}, \Delta t_{m}} \underset{m \rightarrow+\infty}{\longrightarrow} \xi(p) \quad \text { weakly in } L^{2}\left(\left(0, t_{f}\right) ; H^{1}(\Omega)\right) .
\end{aligned}
$$

Proof. Thanks to (33), the sequence $\left(\nabla \xi_{\mathcal{T}_{m}, \Delta t_{m}}\right)_{m \geq 1}$ is bounded in $\left(L^{2}\left(Q_{t_{f}}\right)\right)^{2}$. Moreover, it follows from (39) that $\left(\xi_{\mathcal{T}_{m}, \Delta t_{m}}\right)_{m \geq 1}$ is uniformly bounded in $L^{2}\left(Q_{t_{f}}\right)$, providing the boundedness of $\left(\xi_{\mathcal{T}_{m}, \Delta t_{m}}\right)_{m \geq 1}$ in $L^{2}\left(\left(0, t_{f}\right) ; H^{1}(\Omega)\right)$. Therefore, there exists $\Xi \in L^{2}\left(\left(0, t_{f}\right) ; H^{1}(\Omega)\right)$ such that

$$
\xi_{\mathcal{T}_{m}, \Delta t_{m}} \underset{m \rightarrow+\infty}{\longrightarrow} \Xi \quad \text { weakly in } L^{2}\left(\left(0, t_{f}\right) ; H^{1}(\Omega)\right) .
$$

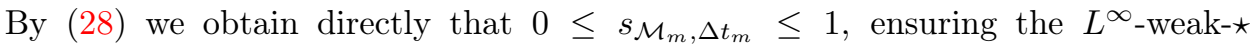
convergence of an unlabeled subsequence towards $s \in L^{\infty}\left(Q_{t_{f}} ;[0,1]\right)$. Thanks to Lemma 4.1, we can apply [4, Theorem 3.9]. It gives the existence of $p: Q_{t_{f}} \longrightarrow$ $\left[p_{\star}, p^{\star}\right]$ such that, up to an unlabeled subsequence,

$$
s_{\mathcal{M}_{m}, \Delta t_{m}}^{\longrightarrow} \underset{m \rightarrow+\infty}{\longrightarrow} s(p) \text { a.e in } Q_{t_{f}}
$$

and $\Xi=\xi(p)$. 


\subsection{Identification as a weak solution.}

Proposition 4.3. Let $p$ be as in Proposition 4.2, then $p$ is the unique weak solution to (1) in the sense of Definition 1.2.

Proof. Let $\psi \in C_{c}^{\infty}\left(\bar{\Omega} \times\left[0, t_{f}\right)\right)$, and denote by $\psi_{K}^{n}=\psi\left(\boldsymbol{x}_{K}, t_{n}\right)$, for all $K \in \mathcal{V}_{m}$ and all $n \in\left\{0, \ldots, N_{m}\right\}$. We multiply (20a) by $\Delta t_{m} \psi_{K}^{n}$ and sum over $n \in\left\{0, \ldots, N_{m}\right\}$ and $K \in \mathcal{V}_{m}$ to obtain

$$
A_{m}+B_{m}+C_{m}+D_{m}=0
$$

where, denoting by $\xi_{K}^{n+1}=\xi\left(p_{K}^{n+1}\right)$, we have set

$$
\begin{aligned}
A_{m} & =\sum_{n=0}^{N_{m}} \sum_{K \in \mathcal{V}_{m}}\left(s_{K}^{n+1}-s_{K}^{n}\right) \psi_{K}^{n} m_{K}, \\
B_{m} & =\sum_{n=0}^{N_{m}} \Delta t_{m} \sum_{\sigma_{K L} \in \mathcal{E}_{m}} a_{K L}\left(\eta_{K L}^{n+1}\left(p_{K}^{n+1}-p_{L}^{n+1}\right)-\sqrt{\eta_{K L}^{n+1}}\left(\xi_{K}^{n+1}-\xi_{L}^{n+1}\right)\right)\left(\psi_{K}^{n}-\psi_{L}^{n}\right), \\
C_{m} & =\sum_{n=0}^{N_{m}} \Delta t_{m} \sum_{\sigma_{K L} \in \mathcal{E}_{m}} a_{K L} \sqrt{\eta_{K L}^{n+1}}\left(\xi_{K}^{n+1}-\xi_{L}^{n+1}\right)\left(\psi_{K}^{n}-\psi_{L}^{n}\right), \\
D_{m}= & \sum_{n=0}^{N_{m}} \Delta t_{m} \sum_{\sigma_{K L} \in \mathcal{E}_{m}} a_{K L} \eta_{K L}^{n+1}\left(z_{K}-z_{L}\right)\left(\psi_{K}^{n}-\psi_{L}^{n}\right) .
\end{aligned}
$$

Note that $\psi_{K}^{N_{m}+1}=0$ for all $K \in \mathcal{V}_{m}$, then a discrete integration parts yields

$$
\begin{aligned}
A_{m} & =-\sum_{n=0}^{N_{m}} \Delta t_{m} \sum_{K \in \mathcal{V}_{m}} s_{K}^{n+1} \frac{\psi_{K}^{n+1}-\psi_{K}^{n}}{\Delta t_{m}} m_{K}-\sum_{K \in \mathcal{V}_{m}} s_{K}^{0} \psi_{K}^{0} m_{K} \\
& =-\iint_{Q_{t_{f}}} s_{\mathcal{M}_{m}, \Delta t_{m}} \delta \psi_{\mathcal{M}_{m}, \Delta t_{m}} \mathrm{~d} \boldsymbol{x} \mathrm{d} t-\int_{\Omega} s_{\mathcal{M}_{m}}^{0} \psi_{\mathcal{M}_{m}, \Delta t_{m}}(\boldsymbol{x}, 0) \mathrm{d} \boldsymbol{x},
\end{aligned}
$$

where the function $\delta \psi_{\mathcal{M}_{m}, \Delta t_{m}}$ of $X_{\mathcal{M}_{m}, \Delta t_{m}}$ is defined by

$$
\delta \psi_{\mathcal{M}_{m}, \Delta t_{m}}(\boldsymbol{x}, t)=\frac{\psi_{K}^{n+1}-\psi_{K}^{n}}{\Delta t_{m}} \quad \text { if }(\boldsymbol{x}, t) \in \omega_{K} \times\left(t_{n}, t_{n+1}\right) .
$$

Thanks to the regularity of $\psi$, the function $\delta \psi_{\mathcal{M}_{m}, \Delta t_{m}}$ converges uniformly towards $\partial_{t} \psi$ on $Q_{t_{f}}$. Moreover, we have

$$
s_{\mathcal{M}_{m}, \Delta t_{m}} \longrightarrow s(p) \quad \text { in } L^{r}\left(Q_{t_{f}}\right) \text { as } m \rightarrow \infty,
$$

for all $r \in[1, \infty)$ thanks to Proposition 4.2. Therefore,

$$
\iint_{Q_{t_{f}}} s_{\mathcal{M}_{m}, \Delta t_{m}} \delta \psi_{\mathcal{M}_{m}, \Delta t_{m}} \mathrm{~d} \boldsymbol{x} \mathrm{d} t \longrightarrow \iint_{Q_{t_{f}}} s(p) \partial_{t} \psi \mathrm{d} \boldsymbol{x} \mathrm{d} t \quad \text { as } m \rightarrow \infty .
$$

Moreover, $s_{\mathcal{M}_{m}}^{0}$ converges strongly in $L^{1}(\Omega)$ towards the initial data $s_{0}$ and $\psi_{\mathcal{M}_{m}, \Delta t_{m}}(\cdot, 0)$ converges uniformly towards $\psi(\cdot, 0)$. Therefore, we get that

$$
\int_{\Omega} s_{\mathcal{M}_{m}}^{0}(\boldsymbol{x}) \psi_{\mathcal{M}_{m}, \Delta t_{m}}(\boldsymbol{x}, 0) \mathrm{d} \boldsymbol{x} \longrightarrow \int_{\Omega} s_{0}(\boldsymbol{x}) \psi(\boldsymbol{x}, 0) \mathrm{d} \boldsymbol{x} \quad \text { as } m \rightarrow \infty .
$$

We deduce from (47) and (48) that

$$
A_{m} \longrightarrow-\iint_{Q_{t_{f}}} s(p) \partial_{t} \psi \mathrm{d} \boldsymbol{x} \mathrm{d} t-\int_{\Omega} s_{0} \psi(., 0) \mathrm{d} \boldsymbol{x} \quad \text { as } m \rightarrow \infty
$$


The term $B_{m}$ rewrites

$$
B_{m}=\sum_{n=0}^{N_{m}} \Delta t_{m} \sum_{\sigma_{K L} \in \mathcal{E}_{m}} a_{K L} \sqrt{\eta_{K L}^{n+1}}\left(\sqrt{\eta_{K L}^{n+1}}-\sqrt{\tilde{\eta}_{K L}^{n+1}}\right)\left(p_{K}^{n+1}-p_{L}^{n+1}\right)\left(\psi_{K}^{n}-\psi_{L}^{n}\right),
$$

where $\tilde{\eta}_{K L}^{n+1}$ is defined by (34). Using the Cauchy-Schwarz inequality, we get

$$
\begin{aligned}
\left|B_{m}\right|^{2} \leq & \left(\sum_{n=0}^{N_{m}} \Delta t_{m} \sum_{\sigma_{K L} \in \mathcal{E}_{m}}\left|a_{K L}\right| \eta_{K L}^{n+1}\left(p_{K}^{n+1}-p_{L}^{n+1}\right)^{2}\right) \\
& \times \underbrace{\left(\sum_{n=0}^{N_{m}} \Delta t_{m} \sum_{\sigma_{K L} \in \mathcal{E}_{m}}\left|a_{K L}\right|\left(\sqrt{\eta_{K L}^{n+1}}-\sqrt{\tilde{\eta}_{K L}^{n+1}}\right)^{2}\left(\psi_{K}^{n}-\psi_{L}^{n}\right)^{2}\right)}_{:=R_{m}} .
\end{aligned}
$$

The first term in the right-hand side of (50) is bounded by $C_{8}$ thanks to Lemma 3.6. Therefore, in order to prove that $\lim _{m \rightarrow \infty} B_{m}=0$, it suffices to prove that $\lim _{m \rightarrow \infty} R_{m}=$ 0 . For $T \in \mathcal{T}_{m}$, we denote by

$$
\bar{\xi}_{T}^{n+1}=\max _{\boldsymbol{x} \in T}\left(\xi_{\mathcal{T}_{m}, \Delta t_{m}}\left(\boldsymbol{x}, t_{n+1}\right)\right), \quad \underline{\xi}_{T}^{n+1}=\min _{\boldsymbol{x} \in T}\left(\xi_{\mathcal{T}_{m}, \Delta t_{m}}\left(\boldsymbol{x}, t_{n+1}\right)\right),
$$

and we define the piecewise constant functions $\bar{\xi}_{\mathcal{T}_{m}, \Delta t_{m}}$ and $\underline{\xi}_{\mathcal{T}_{m}, \Delta t_{m}}$ by

$$
\bar{\xi}_{\mathcal{T}_{m}, \Delta t_{m}}(\boldsymbol{x}, t)=\bar{\xi}_{T}^{n+1} \quad \text { and } \quad \underline{\xi}_{\mathcal{T}_{m}, \Delta t_{m}}(\boldsymbol{x}, t)=\underline{\xi}_{T}^{n+1} \quad \text { if }(\boldsymbol{x}, t) \in T \times\left(t_{n}, t_{n+1}\right),
$$

Since $\sqrt{\eta \circ s \circ \xi^{-1}}$ is uniformly continuous, it admits a modulus of continuity $\mu$ such that $\mu(0)=0$. Therefore, we can estimate

$$
\left|\sqrt{\eta_{K L}^{n+1}}-\sqrt{\tilde{\eta}_{K L}^{n+1}}\right| \leq \mu\left(\bar{\xi}_{T}^{n+1}-\underline{\xi}_{T}^{n+1}\right), \quad \forall \sigma_{K L} \in \mathcal{E}_{T}
$$

Using (51) in the definition (50) of $R_{m}$, we get

$$
0 \leq R_{m} \leq \sum_{n=0}^{N_{m}} \Delta t_{m} \sum_{T \in \mathcal{T}_{m}} \mu\left(\bar{\xi}_{T}^{n+1}-\underline{\xi}_{T}^{n+1}\right)^{2} \sum_{\sigma_{K L} \in \mathcal{E}_{T}}\left|a_{K L}^{T}\right|\left(\psi_{K}^{n}-\psi_{L}^{n}\right)^{2} .
$$

Following the proof of Lemma 2.1 (cf. [9, Lemma 3.2]), we can prove that

$$
\sum_{\sigma_{K L} \in \mathcal{E}_{T}}\left|a_{K L}^{T}\right|\left(\psi_{K}^{n}-\psi_{L}^{n}\right)^{2} \leq C_{3} \bar{\Lambda}\|\nabla \psi\|_{\infty}^{2} \operatorname{meas}(T), \quad \forall T \in \mathcal{T} .
$$

Therefore, we deduce from (52) that

$$
0 \leq R_{m} \leq C \iint_{Q_{t_{f}}} \mu\left(\bar{\xi}_{\mathcal{T}_{m}, \Delta t_{m}}-\underline{\xi}_{\mathcal{T}_{m}, \Delta t_{m}}\right)^{2} \mathrm{~d} \boldsymbol{x} \mathrm{d} t
$$

where $C$ depends only on $\Lambda, \theta$ and $\psi$. Since $\mu$ is bounded (as $\eta$ is bounded), continuous, with $\mu(0)=0$, it suffices to show that $\bar{\xi}_{\mathcal{T}_{m}, \Delta t_{m}}(\boldsymbol{x}, t)-\underline{\xi}_{\mathcal{T}_{m}, \Delta t_{m}}(\boldsymbol{x}, t)$ tends to 0 almost everywhere in $Q_{t_{f}}$ as $m \rightarrow \infty$ (up to an unlabeled subsequence). Thanks to [9, Lemma A.1] and to Lebesgue's dominated convergence theorem, one 
has

$$
\begin{aligned}
& \iint_{Q_{t_{f}}}\left|\bar{\xi}_{\mathcal{T}_{m}, \Delta t_{m}}(\boldsymbol{x}, t)-\underline{\xi}_{\mathcal{T}_{m}, \Delta t_{m}}(\boldsymbol{x}, t)\right| \mathrm{d} \boldsymbol{x} \mathrm{d} t \\
& \leq C h_{m} \iint_{Q_{t_{f}}}\left|\nabla \xi_{\mathcal{T}_{m}, \Delta t_{m}}\right| \mathrm{d} \boldsymbol{x} \mathrm{d} t \underset{m \rightarrow+\infty}{\longrightarrow} 0 .
\end{aligned}
$$

As a consequence of (50), (54) and (55), and still up to the extraction of an unlabeled subsequence, one has

$$
\lim _{m \rightarrow \infty} B_{m}=\lim _{m \rightarrow \infty} R_{m}=0 .
$$

Let us now focus on the term $C_{m}$. We define the piecewise constant functions $\Xi_{\mathcal{T}_{m}, \Delta t_{m}}$ and $H_{\mathcal{T}_{m}, \Delta t_{m}}$ by

$$
\Xi_{\mathcal{T}_{m}, \Delta t_{m}}(\boldsymbol{x}, t)=\xi_{\mathcal{T}_{m}, \Delta t_{m}}\left(\boldsymbol{x}_{T}, t\right), \quad \forall \boldsymbol{x} \in T, \forall t \in\left(t_{n}, t_{n+1}\right),
$$

$\boldsymbol{x}_{T}$ being the center of mass of the triangle $T$, and by $H_{\mathcal{T}_{m}, \Delta t_{m}}=\eta \circ s \circ \xi^{-1}\left(\Xi_{\mathcal{T}_{m}, \Delta t_{m}}\right)$. Clearly, one has

$$
\underline{\xi}_{\mathcal{T}_{m}, \Delta t_{m}} \leq \Xi_{\mathcal{T}_{m}, \Delta t_{m}} \leq \bar{\xi}_{\mathcal{T}_{m}, \Delta t_{m}}
$$

It follows from (55) that both $\underline{\xi}_{\mathcal{T}_{m}, \Delta t_{m}}$ and $\bar{\xi}_{\mathcal{T}_{m}, \Delta t_{m}}$ converge almost everywhere to $\xi(p)$, hence so does $\Xi_{\mathcal{T}_{m}, \Delta t_{m}}$. This provides that

$$
H_{\mathcal{T}_{m}, \Delta t_{m}} \longrightarrow \eta(s(p)) \quad \text { in } L^{1}\left(Q_{t_{f}}\right) \text { as } m \rightarrow \infty .
$$

We introduce the term

$$
C_{m}^{\prime}=\iint_{Q_{t_{f}}} \sqrt{H_{\mathcal{T}_{m}, \Delta t_{m}}} \Lambda \nabla \xi_{\mathcal{T}_{m}, \Delta t_{m}} \cdot \nabla \psi_{\mathcal{T}_{m}, \Delta t_{m}}\left(., t-\Delta t_{m}\right) \mathrm{d} \boldsymbol{x} \mathrm{d} t .
$$

Since $\nabla \xi_{\mathcal{T}_{m}, \Delta t_{m}}$ converges weakly in $L^{2}\left(Q_{t_{f}}\right)$ towards $\nabla \xi(p)$, since $\nabla \psi_{\mathcal{T}_{m}, \Delta t_{m}}$ converges uniformly towards $\nabla \psi$, and thanks to (57), we obtain that

$$
\lim _{m \rightarrow \infty} C_{m}^{\prime}=\iint_{Q_{t_{f}}} \sqrt{\eta(s(p))} \Lambda \nabla \xi(p) \cdot \nabla \psi \mathrm{d} \boldsymbol{x} \mathrm{d} t
$$

Let us now check that $\left|C_{m}-C_{m}^{\prime}\right|$ tends to 0 as $m$ tends to $\infty$. We denote by

$$
\eta_{T}^{n+1}=H_{\mathcal{T}_{m}, \Delta t_{m}}\left(\boldsymbol{x}_{T}, t_{n+1}\right), \quad \forall T \in \mathcal{T}_{m}, \forall n \in\left\{0, \ldots, N_{m}\right\} .
$$

The term $C_{m}^{\prime}$ can be rewritten

$$
C_{m}^{\prime}=\sum_{n=0}^{N_{m}} \Delta t_{m} \sum_{T \in \mathcal{T}_{m}} \sqrt{\eta_{T}^{n+1}} \sum_{\sigma_{K L} \in \mathcal{E}_{T}} a_{K L}^{T}\left(\xi_{K}^{n+1}-\xi_{L}^{n+1}\right)\left(\psi_{K}^{n}-\psi_{L}^{n}\right),
$$

so that

$C_{m}-C_{m}^{\prime}=\sum_{n=0}^{N_{m}} \Delta t_{m} \sum_{T \in \mathcal{T}_{m}} \sum_{\sigma_{K L} \in \mathcal{E}_{T}} a_{K L}^{T}\left(\sqrt{\eta_{K L}^{n+1}}-\sqrt{\eta_{T}^{n+1}}\right)\left(\xi_{K}^{n+1}-\xi_{L}^{n+1}\right)\left(\psi_{K}^{n}-\psi_{L}^{n}\right)$.

For all $n \in\left\{0, \ldots, N_{m}\right\}$, for all $T \in \mathcal{T}_{m}$, and for all $\sigma_{K L} \in \mathcal{E}_{T}$, one has

$$
\left|\sqrt{\eta_{K L}^{n+1}}-\sqrt{\eta_{T}^{n+1}}\right| \leq \mu\left(\bar{\xi}_{T}^{n+1}-\underline{\xi}_{T}^{n+1}\right)
$$


where $\mu$ is the continuity modulus of $\sqrt{\eta \circ s \circ \xi^{-1}}$. Then one obtains that

$$
\begin{aligned}
\left|C_{m}-C_{m}^{\prime}\right| \leq \sum_{n=0}^{N_{m}} \Delta t_{m} \sum_{T \in \mathcal{T}_{m}}\left[\mu\left(\bar{\xi}_{T}^{n+1}-\underline{\xi}_{T}^{n+1}\right)\right. & \\
& \left.\times \sum_{\sigma_{K L} \in \mathcal{E}_{T}}\left|a_{K L}^{T}\right|\left|\xi_{K}^{n+1}-\xi_{L}^{N+1}\right|\left|\psi_{K}^{n}-\psi_{L}^{n}\right|\right] .
\end{aligned}
$$

The Cauchy-Schwarz inequality provides

$$
\begin{aligned}
\left|C_{m}-C_{m}^{\prime}\right|^{2} \leq \sum_{n=0}^{N_{m}} \Delta t_{m} \sum_{T \in \mathcal{T}_{m}} \mu & \left(\bar{\xi}_{T}^{n+1}-\underline{\xi}_{T}^{n+1}\right)^{2} \sum_{\sigma_{K L} \in \mathcal{E}_{T}}\left|a_{K L}^{T}\right|\left(\psi_{K}^{n}-\psi_{L}^{n}\right)^{2} \\
& \times \sum_{n=0}^{N_{m}} \Delta t_{m} \sum_{T \in \mathcal{T}_{m}} \sum_{\sigma_{K L} \in \mathcal{E}_{T}}\left|a_{K L}^{T}\right|\left(\xi_{K}^{n+1}-\xi_{L}^{n+1}\right)^{2} .
\end{aligned}
$$

Using Lemma 2.1 and Lemma 3.4, together with (53), one deduces that

$$
\left|C_{m}-C_{m}^{\prime}\right|^{2} \leq C \iint_{Q_{t_{f}}} \mu\left(\bar{\xi}_{\mathcal{T}_{m}, \Delta t_{m}}-\underline{\xi}_{\mathcal{T}_{m}, \Delta t_{m}}\right)^{2} \mathrm{~d} \boldsymbol{x} \mathrm{d} t
$$

thus $\left|C_{m}-C_{m}^{\prime}\right|$ tends to 0 thanks to the arguments already developed to prove that $R_{m}$ tends to 0 . Finally, we obtain that

$$
\lim _{m \rightarrow \infty} C_{m}=\iint_{Q_{t_{f}}} \sqrt{\eta(s(p))} \Lambda \nabla \xi(p) \cdot \nabla \psi \mathrm{d} \boldsymbol{x} \mathrm{d} t
$$

Let us focus on the last term $D_{m}$. We introduce the term

$$
D_{m}^{\prime}=\iint_{Q_{t_{f}}} H_{\mathcal{T}_{m}, \Delta t_{m}} \Lambda \nabla z \cdot \nabla \psi_{\mathcal{T}_{m}, \Delta t_{m}}\left(., t-\Delta t_{m}\right) \mathrm{d} \boldsymbol{x} \mathrm{d} t
$$

It follows from (57) and from the uniform convergence of $\nabla \psi_{\mathcal{T}_{m}, \Delta t_{m}}$ towards $\nabla \psi$ as $m$ tends to $+\infty$ that

$$
\lim _{m \rightarrow \infty} D_{m}^{\prime}=\iint_{Q_{t_{f}}} \eta(s(p)) \Lambda \nabla z \cdot \nabla \psi \mathrm{d} \boldsymbol{x} \mathrm{d} t
$$

We will now check that $\left|D_{m}-D_{m}^{\prime}\right| \longrightarrow 0$ as $m \rightarrow \infty$. The term $D_{m}^{\prime}$ rewrites

$$
D_{m}^{\prime}=\sum_{n=0}^{N_{m}} \Delta t_{m} \sum_{T \in \mathcal{T}_{m}} \eta_{T}^{n+1} \sum_{\sigma_{K L} \in \mathcal{E}_{T}} a_{K L}^{T}\left(z_{K}-z_{L}\right)\left(\psi_{K}^{n}-\psi_{L}^{n}\right),
$$

so that

$$
D_{m}-D_{m}^{\prime}=\sum_{n=0}^{N_{m}} \Delta t_{m} \sum_{T \in \mathcal{T}_{m}} \sum_{\sigma_{K L} \in \mathcal{E}_{T}} a_{K L}^{T}\left(\eta_{K L}^{n+1}-\eta_{T}^{n+1}\right)\left(z_{K}-z_{L}\right)\left(\psi_{K}^{n}-\psi_{L}^{n}\right) .
$$

For all $\sigma_{K L} \in \mathcal{E}_{T}$, one has

$$
\left|\eta_{K L}^{n+1}-\eta_{T}^{n+1}\right| \leq\left|\sqrt{\eta_{K L}^{n+1}}-\sqrt{\eta_{T}^{n+1}}\right|\left(\sqrt{\eta_{K L}^{n+1}}+\sqrt{\eta_{T}^{n+1}}\right) \leq 2\|\eta\|_{\infty} \mu\left(\bar{\xi}_{T}^{n+1}-\underline{\xi}_{T}^{n+1}\right) .
$$


Therefore, using the Cauchy-Schwarz inequality, one has

$$
\begin{aligned}
\left|D_{m}-D_{m}^{\prime}\right|^{2} \leq & 4\|\eta\|_{\infty}^{2} \sum_{n=0}^{N_{m}} \Delta t_{m} \sum_{T \in \mathcal{T}_{m}} \mu\left(\bar{\xi}_{T}^{n+1}-\underline{\xi}_{T}^{n+1}\right)^{2} \sum_{\sigma_{K L} \in \mathcal{E}_{T}}\left|a_{K L}^{T}\right|\left(\psi_{K}^{n}-\psi_{L}^{n}\right)^{2} \\
& \times \sum_{n=0}^{N_{m}} \Delta t_{m} \sum_{T \in \mathcal{T}_{m}} \sum_{\sigma_{K L} \in \mathcal{E}_{T}}\left|a_{K L}^{T}\right|\left(z_{K}-z_{L}\right)^{2}
\end{aligned}
$$

We use Lemma 2.1 to get

$$
\sum_{n=0}^{N_{m}} \Delta t_{m} \sum_{T \in \mathcal{T}_{m}} \sum_{\sigma_{K L} \in \mathcal{E}_{T}}\left|a_{K L}^{T}\right|\left(z_{K}-z_{L}\right)^{2} \leq C_{3} \iint_{Q_{t_{f}}} \Lambda \nabla z \cdot \nabla z \mathrm{~d} \boldsymbol{x} \mathrm{d} t \leq C .
$$

We deduce from (53) that

$$
\left|D_{m}-D_{m}^{\prime}\right|^{2} \leq C \iint_{Q_{t_{f}}} \mu\left(\bar{\xi}_{\mathcal{T}_{m}, \Delta t_{m}}-\underline{\xi}_{\mathcal{T}_{m}, \Delta t_{m}}\right)^{2} \mathrm{~d} \boldsymbol{x} \mathrm{d} t \underset{m \rightarrow \infty}{\longrightarrow} 0,
$$

and then that

$$
\lim _{m \rightarrow \infty} D_{m}=\iint_{Q_{t_{f}}} \eta(s(p)) \Lambda \nabla z \cdot \nabla \psi \mathrm{d} \boldsymbol{x} \mathrm{d} t .
$$

Putting (49), (56), (60) and (61) together in (46) provides that p satisfies the weak formulation (12), then it is the unique weak solution to the problem (cf. Theorem 1.3).

Finally, let us remark that since the weak solution $p$ is unique, all the convergence in functional space that were proved to occur up to the extraction of a subsequence are valid for the whole sequences. Concerning the almost everywhere convergence, we cannot do better than saying that it holds up to a subsequence.

\section{Numerical RESUlts}

Let us provide some illustrations of the behavior of the numerical scheme (20). The scheme leads to a nonlinear system that we solve thanks to the NewtonRaphson method with Matlab. In all our test cases, the domain is the unit square, i.e., $\Omega=(0,1)^{2}$. We use meshes coming from the $2 \mathrm{D}$ benchmark on anisotropic diffusion problems [22]. An illustration of the meshes is given in Figure 3. These triangle meshes show no symmetry which could artificially increase the convergence rate. All angles are acute, so that, in the case of an isotropic tensor $\Lambda$, the coefficients $a_{K L}$ are all non-negative. This is no longer the case when $\Lambda$ is chosen to be anisotropic. To be more precise concerning the diffusion tensor, we have considered constant diagonal tensors

$$
\Lambda=\left(\begin{array}{cc}
\Lambda_{x x} & 0 \\
0 & \Lambda_{y y}
\end{array}\right)
$$

where $\Lambda_{x x}$ and $\Lambda_{y y}$ are chosen constant in $\Omega$, and the gravity acceleration $\mathbf{g}$ is defined by $\mathbf{g}=(g, 0)^{T}$ for all $\boldsymbol{x} \in \Omega$ with $g \in \mathbb{R}_{+}$.

The numerical analysis of the scheme was carried out for a uniform time discretization of $\left(0, t_{f}\right)$ only in order to avoid heavy notations. In order to increase the robustness of the algorithm and to ensure the convergence of the Newton-Raphson iterative procedure, we used an adaptive time step procedure in the practical implementation. More precisely, to each mesh, we associate a maximal time step $\Delta t_{k}$, 

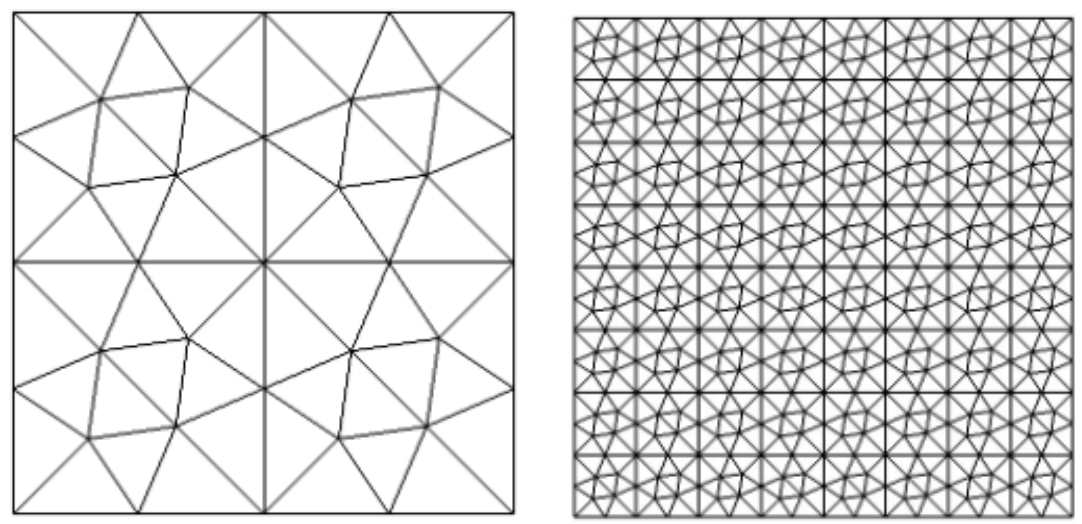

Figure 3. Second and fourth meshes used in the numerical tests

$k$ being the index of the mesh ( 1 for the coarsest, 7 for the finest). If the NewtonRaphson method fails to converge after 30 iterations, the time step is divided by two. If the Newton-Raphson method converges, the time step is multiplied by two and projected on $\left[0, \Delta t_{k}\right]$.

In sections $\S 5.1, \S 5.2$ and $\S 5.3$, we give evidence of the convergence of scheme (20) on test cases where exact analytical solutions are known. We are interested in the convergence speed of our method when the discretization parameters $h$ and $\Delta t$ tend to 0 . We focus on the error caused by the spatial discretization (the time discretization is a classical first order accurate backward Euler method). As we will see, our scheme is at most first order accurate. In order to be sure that the error caused by the time discretization will not be of leading order, we choose $\Delta t_{k+1}=\Delta t_{k} / 4$ while $h_{k+1}=h_{k} / 2, h_{k}$ being the size the mesh $k \in\{1, \ldots, 7\}$. The first time step $\Delta t_{1}$ to 0.01024 in all the test cases presented below.

The test cases we chose to present here do not perfectly match with the assumptions presented at the beginning of the paper. They rather isolate the difficulties of the problem and give a better view of the behavior of the scheme. More precisely, the so called Hornung-Messing problem presented in $\S 5.1$ aims to illustrate the behavior of the scheme when an elliptic degeneracy occurs. The linear FokkerPlanck problem of $\S 5.2$ illustrates the behavior of the scheme for a stiff problem when $p_{\star}=-\infty$. The porous medium equation with drift presented in $\S 5.3$ allows to illustrate the behavior of the scheme near a hyperbolic degeneracy at $s(p)=0$. Finally, the last test case presented in $§ 5.4$ is there to illustrate numerically the decay of the free energy. Let us stress that the numerical analysis we developed in the paper can be adapted without any major modification to all the cases we present here.

Let us mention that in the tests 2,3 , and 4 , we considered problems without elliptic degeneracy. The corresponding functions $s$ are increasing on $\left(p_{\star},+\infty\right)$. Therefore, we can choose $S=s(p)$ rather that $p$ as a primary unknown in these cases. Denoting by $p=s^{-1}$, the problem solved numerically in $\S 5.2, \S 5.3$ and $\S 5.4$ can then be written

$$
\partial_{t} S-\nabla \cdot(\Lambda \eta(S)(\nabla p(S)-\mathbf{g}))=0 \quad \text { in } Q_{t_{f}} .
$$


Finally, we have set the gravity $\mathbf{g}=\mathbf{e}_{x}$ horizontal from the left to the right in the tests 2,3 , and 4 . As a consequence, the scheme we considered in $\S 5.2, \S 5.3$, and $\S 5.4$ is

$$
\begin{aligned}
& \frac{s_{K}^{n+1}-s_{K}^{n}}{\Delta t} m_{K}+\sum_{\sigma_{K L} \in \mathcal{E}_{K}} \eta_{K L}^{n+1} a_{K L}\left(u_{K}^{n+1}-u_{L}^{n+1}\right)=0, \\
& u_{K}^{n+1}=p_{K}^{n+1}-x_{K}, \\
& p_{K}^{n+1}=p\left(s_{K}^{n+1}\right), \\
& \eta_{K L}^{n+1}=\left\{\begin{array}{lll}
\eta\left(s_{K}^{n+1}\right) & \text { if } & a_{K L}\left(u_{K}^{n+1}-u_{L}^{n+1}\right) \geq 0 \\
\eta\left(s_{L}^{n+1}\right) & \text { if } & a_{K L}\left(u_{K}^{n+1}-u_{L}^{n+1}\right)<0 .
\end{array}\right.
\end{aligned}
$$

5.1. Test 1: A test case with saturated zones. The first test-case we propose here is the so-called Hornung-Messing problem [7]. In this problem, gravity is neglected (i.e. $g=0$ and $u_{K}^{n+1}=p_{K}^{n+1}$ for all $K \in \mathcal{V}$ and $n \geq 0$ ). We consider the following nonlinearities

$$
\eta(p)=\left\{\begin{array}{ll}
\frac{2}{1+p^{2}} & \text { if } p<0, \\
2 & \text { if } p \geq 0,
\end{array} \quad s(p)= \begin{cases}\left(\frac{\pi^{2}}{4}-\arctan ^{2}(p)\right)\left(\Lambda_{x x}+\Lambda_{y y}\right) & \text { if } p<0, \\
\frac{\pi^{2}}{4}\left(\Lambda_{x x}+\Lambda_{y y}\right) & \text { if } p \geq 0 .\end{cases}\right.
$$

and the exact solution to the Richards equation

(64) $p_{\mathrm{ex}}=\left\{\begin{array}{ll}-\frac{x-y-t}{2} & \text { if } x-y-t<0, \\ -\tan \left(\frac{e^{x-y-t}-1}{e^{x-y-t}+1}\right) & \text { if } x-y-t \geq 0,\end{array} \quad \forall(x, y) \in \Omega, \forall t \in\left(0, t_{f}\right)\right.$,

where $t_{f}$ was set to 0.05 . This exact solution does not satisfies the no-flux boundary conditions. Therefore, we prescribe the exact solution $p_{\text {ex }}$ as Dirichlet boundary conditions on $\partial \Omega \times\left(0, t_{f}\right)$. In Tables 1 and 2 , we report the errors

$$
\operatorname{err}_{L^{p}}=\left\|p_{\mathcal{M}, \Delta t}-p_{\mathrm{ex}}\right\|_{L^{p}\left(Q_{t_{f}}\right)} \quad \text { for } p=1,2, \infty
$$

for 7 successively refined meshes in the Isotropic case $\Lambda=\operatorname{Id}$ and in the anisotropic case $\Lambda=\operatorname{diag}\left(1,10^{-3}\right)$.

\begin{tabular}{|lr|lr|lr|lr|}
\hline \multicolumn{1}{|l}{} & $\# \mathcal{V}$ & $\operatorname{err}_{L^{2}}$ & rate & $\operatorname{err}_{L^{1}}$ & rate & $\operatorname{err}_{L^{\infty}}$ & rate \\
\hline 0.500 & 12 & $0.343 \mathrm{E}-3$ & - & $0.548 \mathrm{E}-4$ & - & $0.352 \mathrm{E}-2$ & - \\
0.250 & 37 & $0.218 \mathrm{E}-3$ & 0.651 & $0.472 \mathrm{E}-4$ & 0.215 & $0.197 \mathrm{E}-2$ & 0.838 \\
0.125 & 129 & $0.141 \mathrm{E}-3$ & 0.629 & $0.329 \mathrm{E}-4$ & 0.522 & $0.113 \mathrm{E}-2$ & 0.801 \\
0.063 & 481 & $0.769 \mathrm{E}-4$ & 0.886 & $0.185 \mathrm{E}-4$ & 0.844 & $0.607 \mathrm{E}-3$ & 0.907 \\
0.031 & 1857 & $0.399 \mathrm{E}-4$ & 0.927 & $0.967 \mathrm{E}-5$ & 0.912 & $0.306 \mathrm{E}-3$ & 0.966 \\
0.016 & 7297 & $0.202 \mathrm{E}-4$ & 1.025 & $0.493 \mathrm{E}-5$ & 1.019 & $0.154 \mathrm{E}-3$ & 1.041 \\
0.008 & 28929 & $0.102 \mathrm{E}-4$ & 0.989 & $0.249 \mathrm{E}-5$ & 0.986 & $0.771 \mathrm{E}-4$ & 0.996 \\
0.004 & 115201 & $0.512 \mathrm{E}-5$ & 0.994 & $0.125 \mathrm{E}-5$ & 0.993 & $0.386 \mathrm{E}-4$ & 0.997 \\
\hline
\end{tabular}

TABLE 1 . Test 1 , isotropic case $\Lambda=\mathrm{Id}$.

We observe that numerical order of convergence is close to 1 for the three norms whatever the anisotropy tensor on this test case. 


\begin{tabular}{|lr|lr|lr|lr|}
\hline \multicolumn{1}{l}{$h$} & $\# \mathcal{V}$ & $\operatorname{err}_{L^{2}}$ & rate & $\operatorname{err}_{L^{1}}$ & rate & $\operatorname{err}_{L^{\infty}}$ & rate \\
\hline 0.500 & 12 & $0.382 \mathrm{E}-3$ & - & $0.581 \mathrm{E}-4$ & - & $0.384 \mathrm{E}-2$ & - \\
0.250 & 37 & $0.368 \mathrm{E}-3$ & 0.057 & $0.682 \mathrm{E}-4$ & -0.231 & $0.396 \mathrm{E}-2$ & -0.044 \\
0.125 & 129 & $0.225 \mathrm{E}-3$ & 0.710 & $0.475 \mathrm{E}-4$ & 0.522 & $0.218 \mathrm{E}-2$ & 0.861 \\
0.063 & 481 & $0.120 \mathrm{E}-3$ & 0.911 & $0.268 \mathrm{E}-4$ & 0.838 & $0.112 \mathrm{E}-2$ & 0.974 \\
0.031 & 1857 & $0.621 \mathrm{E}-4$ & 0.933 & $0.141 \mathrm{E}-4$ & 0.904 & $0.522 \mathrm{E}-3$ & 1.075 \\
0.016 & 7297 & $0.315 \mathrm{E}-4$ & 1.026 & $0.721 \mathrm{E}-5$ & 1.012 & $0.260 \mathrm{E}-3$ & 1.052 \\
0.008 & 28929 & $0.159 \mathrm{E}-4$ & 0.990 & $0.365 \mathrm{E}-5$ & 0.983 & $0.130 \mathrm{E}-3$ & 1.003 \\
0.004 & 115201 & $0.796 \mathrm{E}-5$ & 0.993 & $0.183 \mathrm{E}-5$ & 0.992 & $0.647 \mathrm{E}-4$ & 1.002 \\
\hline
\end{tabular}

TABLE 2. Test 1 , anisotropic case with $\Lambda_{x x}=1$ and $\Lambda_{y y}=10^{-3}$

5.2. Test 2: Linear Fokker-Planck equation. In this test case, we study the behavior of our scheme on the problem (1) with the choice of nonlinearities $s(p)=$ $\exp (p)$ and $\eta(s)=s$. The function $s$ does not fulfill Assumption (A1) since $s$ is not constant on $\mathbb{R}_{+}$. Since $s$ in injective, we can use $S=s(p)$ as a primary unknown, leading to the problem

$$
\begin{cases}\partial_{t} S-\nabla \cdot\left(S \Lambda\left(\nabla \log (S)-\mathbf{e}_{x}\right)\right)=0 & \text { in } Q_{t_{f}} \\ S \Lambda\left(\nabla \log (S)-\mathbf{e}_{x}\right) \cdot \mathbf{n}=0 & \text { on } \partial \Omega \times(0, T), \\ S_{\left.\right|_{t=0}}=s_{0} & \text { in } \Omega\end{cases}
$$

that turns out to be the linear convection diffusion equation

$$
\begin{cases}\partial_{t} S-\nabla \cdot\left(\Lambda\left(\nabla S-S \mathbf{e}_{x}\right)=0\right. & \text { in } Q_{t_{f}} \\ \Lambda\left(\nabla S-S \mathbf{e}_{x}\right) \cdot \mathbf{n}=0 & \text { on } \partial \Omega \times(0, T), \\ S_{\left.\right|_{t=0}}=s_{0} & \text { in } \Omega .\end{cases}
$$

We compare the results obtained with the nonlinear CVFE scheme (63) with the following linear scheme where the convection is discretized thanks to centered fluxes:

$$
\frac{s_{K}^{n+1}-s_{K}^{n}}{\Delta t} m_{K}+\sum_{\sigma_{K L} \in \mathcal{E}_{K}} a_{K L}\left(\left(s_{K}^{n+1}-s_{L}^{n+1}\right)+\left(x_{K}-x_{L}\right) \frac{s_{K}^{n+1}+s_{L}^{n+1}}{2}\right)=0
$$

for all $K \in \mathcal{V}$ and for all $n \in\{0, \ldots, N\}$.

The schemes (63) and (67) are compared on the following analytical solution built from a 1D case:

$$
s_{\mathrm{ex}}(x, y, t)=\exp \left(-\alpha t+\frac{x}{2}\right)\left(\pi \cos (\pi x)+\frac{1}{2} \sin (\pi x)\right)+\pi \exp \left(x-\frac{1}{2}\right) \text { in } Q_{t_{f}},
$$

where $\alpha=\Lambda_{x x}\left(\pi^{2}+\frac{1}{4}\right)$, and where the final time has been fixed to 0.05 . This analytical solution is nonnegative and satisfies homogeneous Neumann boundary conditions.

In Tables 3 to 6 , we report the $L^{1}\left(Q_{t_{f}}\right), L^{2}\left(Q_{t_{f}}\right)$, and $L^{\infty}\left(Q_{t_{f}}\right)$ on the variable $S$, i.e.,

$$
e r r_{L^{p}}=\left\|s_{\mathcal{M}, \Delta t}-s_{\mathrm{ex}}\right\|_{L^{p}\left(Q_{t_{f}}\right)} \quad \text { for } p=1,2, \infty
$$

The numerical order of convergence of the linear scheme (67) is close to 2. However, the more the anisotropy ratio is important, the more we observe oscillations and undershoots (see in particular Table 6). The nonlinear scheme (63) preserves 


\begin{tabular}{|lr|lr|lr|lr|c|}
\hline \multicolumn{1}{|l}{$h$} & $\# \mathcal{V}$ & $\operatorname{err}_{L^{2}}$ & rate & err $_{L^{1}}$ & rate & err $_{L^{\infty}}$ & rate & $S_{\min }$ \\
\hline 0.500 & 12 & $0.328 \mathrm{E}-01$ & - & $0.820 \mathrm{E}-02$ & - & $0.232 \mathrm{E}+00$ & - & 0 \\
0.250 & 37 & $0.306 \mathrm{E}-01$ & 0.0389 & $0.798 \mathrm{E}-02$ & -0.0389 & $0.239 \mathrm{E}+00$ & -0.0466 & 0 \\
0.125 & 129 & $0.198 \mathrm{E}-01$ & 0.6320 & $0.508 \mathrm{E}-02$ & 0.6519 & $0.153 \mathrm{E}+00$ & 0.6477 & 0 \\
0.063 & 481 & $0.109 \mathrm{E}-01$ & 0.8674 & $0.276 \mathrm{E}-02$ & 0.8911 & $0.841 \mathrm{E}-01$ & 0.8722 & 0 \\
0.031 & 1857 & $0.570 \mathrm{E}-02$ & 0.9130 & $0.143 \mathrm{E}-02$ & 0.9237 & $0.441 \mathrm{E}-01$ & 0.9101 & 0 \\
0.016 & 7297 & $0.292 \mathrm{E}-02$ & 1.0152 & $0.729 \mathrm{E}-03$ & 1.0214 & $0.226 \mathrm{E}-01$ & 1.0123 & 0 \\
0.008 & 28929 & $0.147 \mathrm{E}-02$ & 0.9845 & $0.368 \mathrm{E}-03$ & 0.9893 & $0.114 \mathrm{E}-01$ & 0.9831 & 0 \\
0.004 & 115201 & $0.741 \mathrm{E}-03$ & 0.9923 & $0.185 \mathrm{E}-03$ & 0.9937 & $0.575 \mathrm{E}-02$ & 0.9913 & 0 \\
\hline
\end{tabular}

TABle 3. Test 2, nonlinear scheme (63), with an isotropic tensor $\Lambda=$ Id.

\begin{tabular}{|lr|lr|lr|lr|c|}
\hline \multicolumn{1}{|l}{} & $\# \mathcal{V}$ & $\operatorname{err}_{L^{2}}$ & rate & $\operatorname{err}_{L^{1}}$ & rate & $\operatorname{err}_{L^{\infty}}$ & rate & $S_{\min }$ \\
\hline 0.500 & 12 & $0.294 \mathrm{E}-01$ & - & $0.372 \mathrm{E}-02$ & - & $0.484 \mathrm{E}+00$ & - & 0 \\
0.250 & 37 & $0.829 \mathrm{E}-02$ & 1.8267 & $0.198 \mathrm{E}-02$ & 1.6352 & $0.166 \mathrm{E}+00$ & 1.5428 & 0 \\
0.125 & 129 & $0.218 \mathrm{E}-02$ & 1.9286 & $0.349 \mathrm{E}-03$ & 1.8389 & $0.426 \mathrm{E}-01$ & 1.9639 & 0 \\
0.063 & 481 & $0.548 \mathrm{E}-03$ & 2.0138 & $0.859 \mathrm{E}-04$ & 1.9863 & $0.108 \mathrm{E}-01$ & 2.0069 & 0 \\
0.031 & 1857 & $0.137 \mathrm{E}-03$ & 1.9521 & $0.216 \mathrm{E}-04$ & 1.9310 & $0.274 \mathrm{E}-02$ & 1.9310 & 0 \\
0.016 & 7297 & $0.343 \mathrm{E}-04$ & 2.0956 & $0.542 \mathrm{E}-05$ & 2.0675 & $0.697 \mathrm{E}-03$ & 2.0675 & 0 \\
0.008 & 28929 & $0.858 \mathrm{E}-05$ & 1.9998 & $0.135 \mathrm{E}-05$ & 1.9994 & $0.178 \mathrm{E}-03$ & 1.9720 & 0 \\
0.004 & 115201 & $0.214 \mathrm{E}-05$ & 2.0000 & $0.339 \mathrm{E}-06$ & 1.9998 & $0.453 \mathrm{E}-04$ & 1.9719 & 0 \\
\hline
\end{tabular}

TABLE 4 . Test 2, linear scheme (67) with an isotropic tensor $\Lambda=\mathrm{Id}$.

\begin{tabular}{|lr|lr|lr|lr|c|}
\hline \multicolumn{1}{l}{$h$} & $\# \mathcal{V}$ & $\operatorname{err}_{L^{2}}$ & rate & $\operatorname{err}_{L^{1}}$ & rate & $\operatorname{err}_{L^{\infty}}$ & rate & $S_{\min }$ \\
\hline 0.500 & 12 & $0.179 \mathrm{E}+00$ & - & $0.488 \mathrm{E}-01$ & - & $1.022 \mathrm{E}+00$ & - & 0 \\
0.250 & 37 & $0.166 \mathrm{E}+00$ & 0.1080 & $0.462 \mathrm{E}-01$ & 0.0792 & $0.959 \mathrm{E}+00$ & 0.0930 & 0 \\
0.125 & 129 & $0.118 \mathrm{E}+00$ & 0.4947 & $0.318 \mathrm{E}-01$ & 0.5396 & $0.744 \mathrm{E}+00$ & 0.3659 & 0 \\
0.063 & 481 & $0.746 \mathrm{E}-01$ & 0.6685 & $0.197 \mathrm{E}-01$ & 0.7008 & $0.504 \mathrm{E}+00$ & 0.5689 & 0 \\
0.031 & 1857 & $0.439 \mathrm{E}-01$ & 0.7498 & $0.113 \mathrm{E}-01$ & 0.7755 & $0.309 \mathrm{E}+00$ & 0.6880 & 0 \\
0.016 & 7297 & $0.243 \mathrm{E}-01$ & 0.8904 & $0.621 \mathrm{E}-02$ & 0.9118 & $0.177 \mathrm{E}+00$ & 0.8416 & 0 \\
0.008 & 28929 & $0.130 \mathrm{E}-01$ & 0.9087 & $0.327 \mathrm{E}-02$ & 0.9229 & $0.964 \mathrm{E}-01$ & 0.8793 & 0 \\
0.004 & 115201 & $0.672 \mathrm{E}-02$ & 0.9481 & $0.169 \mathrm{E}-02$ & 0.9571 & $0.506 \mathrm{E}-01$ & 0.9304 & 0 \\
\hline
\end{tabular}

TABLE 5. Test 2: nonlinear scheme (63) with an anisotropic tensor $\Lambda_{x x}=1$ and $\Lambda_{y y}=20$.

the positivity of the solution whatever the anisotropy, but this property has a cost. Indeed, the numerical diffusion introduced by the nonlinear scheme (63) becomes very important when the anisotropy ratio is large. This yields a loss of accuracy. The method (63) seems to be first order accurate, i.e.,

$$
\operatorname{err}_{L^{p}} \leq C_{p}(\Lambda, \theta) h, \quad p \in\{1,2, \infty\},
$$

but with constants $C_{p}(\Lambda, \theta)$ that strongly depend on the anisotropy ratio and of the regularity of the mesh.

5.3. Test 3: Porous medium equation with drift. In this third test case, we set $s(p)=p / 2$ if $p \geq 0$ and $\eta(s)=s$. Choosing $S=s(p)$ as a primary variable, we 


\begin{tabular}{|lr|lr|lr|lr|c|}
\hline \multicolumn{1}{|c|}{$\boldsymbol{H}$} & $\boldsymbol{N}$ & $\operatorname{err}_{L^{2}}$ & rate & $\operatorname{err}_{L^{1}}$ & rate & $\operatorname{err}_{L^{\infty}}$ & rate & $S_{\min }$ \\
\hline 0.500 & 12 & $0.566 \mathrm{E}-01$ & - & $0.115 \mathrm{E}-01$ & - & $0.376 \mathrm{E}+00$ & - & 0 \\
0.250 & 37 & $0.222 \mathrm{E}-01$ & 1.3523 & $0.427 \mathrm{E}-02$ & 1.4290 & $0.250 \mathrm{E}+00$ & 0.5878 & 0 \\
0.125 & 129 & $0.613 \mathrm{E}-02$ & 1.8553 & $0.119 \mathrm{E}-02$ & 1.8469 & $0.883 \mathrm{E}-01$ & 1.5036 & $-2.1867 \mathrm{E}-03$ \\
0.063 & 481 & $0.155 \mathrm{E}-02$ & 2.0021 & $0.300 \mathrm{E}-03$ & 2.0053 & $0.247 \mathrm{E}-01$ & 1.8621 & $-9.3704 \mathrm{e}-04$ \\
0.031 & 1857 & $0.390 \mathrm{E}-03$ & 1.9506 & $0.755 \mathrm{E}-04$ & 1.9468 & $0.647 \mathrm{E}-02$ & 1.8859 & $-2.6687 \mathrm{e}-04$ \\
0.016 & 7297 & $0.976 \mathrm{E}-04$ & 2.0948 & $0.189 \mathrm{E}-04$ & 2.0952 & $0.168 \mathrm{E}-02$ & 2.0358 & $-6.9729 \mathrm{e}-05$ \\
0.008 & 28929 & $0.244 \mathrm{E}-04$ & 1.9997 & $0.472 \mathrm{E}-05$ & 1.9997 & $0.437 \mathrm{E}-03$ & 1.9470 & $-1.7741 \mathrm{e}-05$ \\
0.004 & 115201 & $0.610 \mathrm{E}-05$ & 1.9999 & $0.118 \mathrm{E}-05$ & 1.9999 & $0.113 \mathrm{E}-03$ & 1.9495 & $-4.4696 \mathrm{e}-06$ \\
\hline
\end{tabular}

TABle 6 . Test 2, linear scheme (67) with an anisotropic tensor:

$\Lambda_{x x}=1$ and $\Lambda_{y y}=20$.

obtain the degenerate parabolic equation

$$
\partial_{t} S-\nabla \cdot\left(S \Lambda\left(2 S-\mathbf{e}_{x}\right)\right)=0 \quad \text { in } Q_{t_{f}},
$$

or equivalently

$$
\partial_{t} S-\nabla \cdot\left(\Lambda\left(\nabla S-\mathbf{e}_{x}\right)\right)=0 \quad \text { in } Q_{t_{f}},
$$

The function $s_{\text {ex }}$ defined by

$$
s_{\mathrm{ex}}(x, y, t)=\max (\beta t-x, 0), \forall((x, y), t) \in Q_{t_{f}}
$$

with $\beta=2 \Lambda_{x x}$ satisfies the equation (69). As in Test 1 , we complement (69) by Dirichlet boundary conditions and an initial condition prescribed by (70). The final time $t_{f}$ has been set to 0.05 .

The nonlinear scheme (63) is adapted to the case of Dirichlet boudary conditions: (63a) is assumed to hold only for $K \in \mathcal{V}_{\text {int }}=\left\{K \in \mathcal{V} \mid \boldsymbol{x}_{K} \notin \partial \Omega\right\}$. The equations (63b) and (63c) are enforced for all $K \in \mathcal{V}$, and (63d) is enforced for all $\sigma_{K L} \in \mathcal{E}_{\text {int }}=\{\sigma \in \mathcal{E} \mid \sigma \not \subset \partial \Omega\}$. In order to close the system, we impose $s_{K}^{n+1}=s_{\mathrm{ex}}\left(\boldsymbol{x}_{K}, t_{n+1}\right)$ for all $K$ such that $\boldsymbol{x}_{K} \in \partial \Omega$.

The numerical results obtained thanks to our scheme are compared with those obtained thanks to a so-called quasilinear scheme where (63a) has been replaced by

$$
\frac{s_{K}^{n+1}-s_{K}^{n}}{\Delta t} m_{K}+\sum_{\sigma_{K L} \in \mathcal{E}} a_{K L}\left(\left(s_{K}^{n+1}\right)^{2}-\left(s_{L}^{n+1}\right)^{2}+\left(x_{K}-x_{L}\right) \frac{s_{K}^{n+1}+s_{L}^{n+1}}{2}\right)=0 .
$$

The analytical solution $s_{\text {ex }}$ defined by (70) belongs to $C\left(\left[0, t_{f}\right), H^{3 / 2-\epsilon}(\Omega)\right)$ for all $\epsilon>0$. Therefore, we expect for the quasilinear scheme (71) a convergence order close to 1.5 in the $L^{2}\left(Q_{t_{f}}\right)$ norm, as observed in Tables 8 and 10.

We observe that, as expected, that the nonlinear scheme (63) has a smaller order of convergence (less than 1) when $\Lambda$ is isotropic, cf. Table 5 . Here again, as in Test 2 , the accuracy is strongly affected by the anisotropy. The numerical diffusion introduced by the scheme increases with the anisotropy ratio. But the solutions to the scheme (20) do not present undershoots, on the contrary to the solutions to the quasilinear scheme (71), cf. 10. In order to illustrate the overdiffusive behavior of the nonlinear scheme (63) as well as the undershoots produced by the quasilinear scheme (71), we present in Figure 4 the snapshots of both numerical solutions at time $t=t_{f}$. 


\begin{tabular}{|lr|lr|lr|lr|r|}
\hline \multicolumn{1}{l}{$h$} & $\# \mathcal{V}$ & $e r r_{L^{2}}$ & rate & $e r r_{L^{1}}$ & rate & $e r r_{L^{\infty}}$ & rate & $S_{\min }$ \\
\hline 0.500 & 12 & $0.174 \mathrm{E}-02$ & - & $0.215 \mathrm{E}-03$ & - & $0.284 \mathrm{E}-01$ & - & 0 \\
0.250 & 37 & $0.238 \mathrm{E}-02$ & -0.4509 & $0.224 \mathrm{E}-03$ & -0.0573 & $0.559 \mathrm{E}-01$ & -0.9751 & 0 \\
0.125 & 129 & $0.168 \mathrm{E}-02$ & 0.5062 & $0.160 \mathrm{E}-03$ & 0.4883 & $0.305 \mathrm{E}-01$ & 0.8754 & 0 \\
0.063 & 481 & $0.100 \mathrm{E}-02$ & 0.7489 & $0.889 \mathrm{E}-04$ & 0.8543 & $0.237 \mathrm{E}-01$ & 0.3644 & $-1.1092 \mathrm{e}-26$ \\
0.031 & 1857 & $0.609 \mathrm{E}-03$ & 0.7049 & $0.486 \mathrm{E}-04$ & 0.8522 & $0.174 \mathrm{E}-01$ & 0.4386 & $-2.5862 \mathrm{e}-23$ \\
0.016 & 7297 & $0.359 \mathrm{E}-03$ & 0.7994 & $0.259 \mathrm{E}-04$ & 0.9509 & $0.113 \mathrm{E}-01$ & 0.6459 & $-2.7142 \mathrm{e}-25$ \\
0.008 & 28929 & $0.206 \mathrm{E}-03$ & 0.8043 & $0.136 \mathrm{E}-04$ & 0.9315 & $0.734 \mathrm{E}-02$ & 0.6301 & $-7.0895 \mathrm{e}-11$ \\
0.004 & 115201 & $0.115 \mathrm{E}-03$ & 0.8445 & $0.703 \mathrm{E}-05$ & 0.9511 & $0.460 \mathrm{E}-02$ & 0.6751 & $-8.4650 \mathrm{e}-11$ \\
\hline
\end{tabular}

TABLE 7. Nonlinear scheme, with an isotropic tensor: $\Lambda_{x x}=1$ and $\Lambda_{y y}=1$

\begin{tabular}{|lr|lr|lr|lr|c|}
\hline \multicolumn{1}{l}{$h$} & $\# \mathcal{V}$ & $\operatorname{err}_{L^{2}}$ & rate & $\operatorname{err}_{L^{1}}$ & rate & $\operatorname{err}_{L^{\infty}}$ & rate & $S_{\min }$ \\
\hline 0.500 & 12 & $0.990 \mathrm{E}-03$ & - & $0.120 \mathrm{E}-03$ & - & $0.166 \mathrm{E}-01$ & - & 0 \\
0.250 & 37 & $0.148 \mathrm{E}-02$ & -0.5805 & $0.129 \mathrm{E}-03$ & -0.1076 & $0.383 \mathrm{E}-01$ & -1.2026 & 0 \\
0.125 & 129 & $0.825 \mathrm{E}-03$ & 0.8427 & $0.720 \mathrm{E}-04$ & 0.8424 & $0.176 \mathrm{E}-01$ & 1.1253 & 0 \\
0.063 & 481 & $0.356 \mathrm{E}-03$ & 1.2265 & $0.268 \mathrm{E}-04$ & 1.4434 & $0.106 \mathrm{E}-01$ & 0.7307 & 0 \\
0.031 & 1857 & $0.151 \mathrm{E}-03$ & 1.2052 & $0.998 \mathrm{E}-05$ & 1.3912 & $0.582 \mathrm{E}-02$ & 0.8507 & 0 \\
0.016 & 7297 & $0.581 \mathrm{E}-04$ & 1.4499 & $0.320 \mathrm{E}-05$ & 1.7193 & $0.296 \mathrm{E}-02$ & 1.0232 & $-1.3853 \mathrm{e}-18$ \\
0.008 & 28929 & $0.214 \mathrm{E}-04$ & 1.4403 & $0.950 \mathrm{E}-06$ & 1.7531 & $0.149 \mathrm{E}-02$ & 0.9921 & $-6.9053 \mathrm{e}-17$ \\
0.004 & 115201 & $0.711 \mathrm{E}-05$ & 1.4722 & $0.270 \mathrm{E}-06$ & 1.8125 & $0.743 \mathrm{E}-03$ & 1.0008 & $-2.1592 \mathrm{e}-18$ \\
\hline
\end{tabular}

TABLE 8. Quasilinear scheme, with an isotropic tensor: $\Lambda_{x x}=1$ and $\Lambda_{y y}=1$

\begin{tabular}{|lr|lr|lr|lr|c|}
\hline \multicolumn{1}{|l}{$h$} & $\# \mathcal{V}$ & $\operatorname{err}_{L^{2}}$ & rate & $\operatorname{err}_{L^{1}}$ & rate & $\operatorname{err}_{L^{\infty}}$ & rate & $S_{\min }$ \\
\hline 0.500 & 12 & $0.672 \mathrm{E}-02$ & - & $0.983 \mathrm{E}-03$ & - & $0.829 \mathrm{E}-01$ & - & 0 \\
0.250 & 37 & $0.664 \mathrm{E}-02$ & 0.0178 & $0.102 \mathrm{E}-02$ & -0.0551 & $0.101 \mathrm{E} 00$ & -0.2802 & 0 \\
0.125 & 129 & $0.552 \mathrm{E}-02$ & 0.2663 & $0.862 \mathrm{E}-03$ & 0.2439 & $0.831 \mathrm{E}-01$ & 0.2774 & $-8.3744 \mathrm{E}-10$ \\
0.063 & 481 & $0.441 \mathrm{E}-02$ & 0.3287 & $0.647 \mathrm{E}-03$ & 0.4191 & $0.699 \mathrm{E}-01$ & 0.2526 & $-2.3267 \mathrm{E}-11$ \\
0.031 & 1857 & $0.345 \mathrm{E}-02$ & 0.3471 & $0.458 \mathrm{E}-03$ & 0.4876 & $0.625 \mathrm{E}-01$ & 0.1586 & $-7.8867 \mathrm{E}-11$ \\
0.016 & 7297 & $0.260 \mathrm{E}-02$ & 0.4284 & $0.310 \mathrm{E}-03$ & 0.5954 & $0.514 \mathrm{E}-01$ & 0.2946 & $-1.6558 \mathrm{E}-10$ \\
0.008 & 28929 & $0.189 \mathrm{E}-02$ & 0.4608 & $0.200 \mathrm{E}-03$ & 0.6241 & $0.409 \mathrm{E}-01$ & 0.3266 & $-1.4031 \mathrm{E}-07$ \\
0.004 & 115201 & $0.132 \mathrm{E}-02$ & 0.5141 & $0.125 \mathrm{E}-03$ & 0.6794 & $0.318 \mathrm{E}-01$ & 0.3666 & $-7.8670 \mathrm{E}-07$ \\
\hline
\end{tabular}

TABle 9. Nonlinear scheme, with an anisotropic tensor: $\Lambda_{x x}=1$ and $\Lambda_{y y}=100$

5.4. Decay of discrete free energy. Let us denote by $\mathfrak{M}\left(Q_{t_{f}}\right)$ the set of the measurable functions mapping $Q_{t_{f}}$ to $\mathbb{R}$. The free energy functional [23] $\mathfrak{E}: \mathfrak{M}\left(Q_{t_{f}}\right) \rightarrow$ $\mathbb{R} \cup\{+\infty\}$, defined by

$$
\mathfrak{E}(p)=\int_{\Omega}(\Gamma(p)+s(p) \mathbf{g} \cdot \boldsymbol{x}) \mathrm{d} \boldsymbol{x}, \quad \forall p \in \mathfrak{M}\left(Q_{t_{f}}\right),
$$

consists in the sum of the capillary energy (6), and the gravitational energy. We have formally the classical energy/dissipation property (10), and in particular $t \longmapsto$ $\mathfrak{E}(p)(t)$ is decreasing. The discrete counterpart of the free energy is

$$
\mathfrak{E}\left(p_{\mathcal{M}}^{n}\right)=\sum_{K \in \mathcal{V}} m_{K}\left[\Gamma\left(p_{K}^{n}\right)+g s\left(p_{K}^{n}\right) x_{K}\right]
$$




\begin{tabular}{|lr|lr|lr|lr|c|}
\hline \multicolumn{1}{l}{$h$} & $\# \mathcal{V}$ & $\operatorname{err}_{L^{2}}$ & rate & $\operatorname{err}_{L^{1}}$ & rate & $\operatorname{err}_{L^{\infty}}$ & rate & $S_{\text {min }}$ \\
\hline 0.500 & 12 & $0.976 \mathrm{E}-02$ & - & $0.159 \mathrm{E}-01$ & - & $0.111 \mathrm{E}+00$ & - & $-5.4034 \mathrm{E}-02$ \\
0.250 & 37 & $0.722 \mathrm{E}-02$ & 0.4337 & $0.110 \mathrm{E}-02$ & 0.5325 & $0.108 \mathrm{E}+00$ & 0.0424 & $-3.5579 \mathrm{E}-02$ \\
0.125 & 129 & $0.414 \mathrm{E}-02$ & 0.8015 & $0.583 \mathrm{E}-03$ & 0.9103 & $0.589 \mathrm{E}-01$ & 0.8736 & $-2.5825 \mathrm{E}-02$ \\
0.063 & 481 & $0.179 \mathrm{E}-02$ & 1.2215 & $0.198 \mathrm{E}-03$ & 1.5786 & $0.419 \mathrm{E}-01$ & 0.4968 & $-1.1696 \mathrm{E}-02$ \\
0.031 & 1857 & $0.779 \mathrm{E}-03$ & 1.1765 & $0.746 \mathrm{E}-03$ & 1.3747 & $0.220 \mathrm{E}-01$ & 0.9062 & $-5.8549 \mathrm{E}-03$ \\
0.016 & 7297 & $0.336 \mathrm{E}-02$ & 1.2698 & $0.262 \mathrm{E}-04$ & 1.5806 & $0.118 \mathrm{E}-01$ & 0.9376 & $-2.9309 \mathrm{E}-03$ \\
0.008 & 28929 & $0.140 \mathrm{E}-03$ & 1.2662 & $0.876 \mathrm{E}-05$ & 1.5822 & $0.636 \mathrm{E}-02$ & 0.8980 & $-1.4663 \mathrm{E}-03$ \\
0.004 & 115201 & $0.565 \mathrm{E}-04$ & 1.3073 & $0.282 \mathrm{E}-05$ & 1.6351 & $0.333 \mathrm{E}-02$ & 0.9341 & $-7.3339 \mathrm{E}-04$ \\
\hline
\end{tabular}

TABLE 10. Quasilinear scheme, with an anisotropic tensor: $\Lambda_{x x}=$ 1 and $\Lambda_{y y}=100$
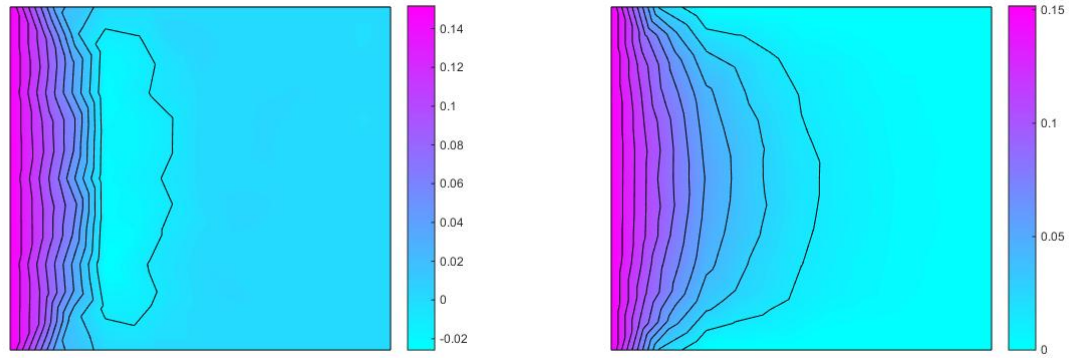

Figure 4. Test 3: 2nd mesh and anisotropic tensor $\Lambda_{x x}=1$ and $\Lambda_{y y}=100$. Discrete solutions $s_{\mathcal{M}, \Delta t}\left(\cdot, t_{f}\right)$ and their iso-values. Left: Quasilinear scheme (71). Right: Nonlinear scheme (63).

We have not succeeded to prove the decay of the discrete free energy contrarily to [9]. Let us provide a numerical evidence of this energy/dissipation property. Define the nonlinearities

$$
s(p)=\left\{\begin{array}{ll}
\frac{1}{1+p^{2}} & \text { if } p<0, \\
1 & \text { if } p \geq 0,
\end{array} \quad \eta(s)=s^{2},\right.
$$

and set $\mathbf{g}=\mathbf{e}_{x}$, and

$$
p_{0}= \begin{cases}-\frac{x-y}{2} & \text { if } x-y<0, \\ -\tan \left(\frac{e^{x-y}-1}{e^{x-y}+1}\right) & \text { if } x-y \geq 0 .\end{cases}
$$

We solve the scheme (20) and we remark (cf. Figure 5) that $\left(\mathfrak{E}\left(p_{\mathcal{M}}^{n}\right)\right)_{n \geq 0}$ is decreasing. As already noticed on the previous test cases, the scheme (20) suffers from an excessive numerical diffusion, in particular when the anisotropy ratio is high. The origins of faster convergence towards the equilibrium in the anisotropic case illustrated by Figure 5 are twofold. The anisotropy favors the convergence towards the equilibrium at the continuous level. But the additional numerical diffusion introduced by the scheme also accelerates this convergence. 

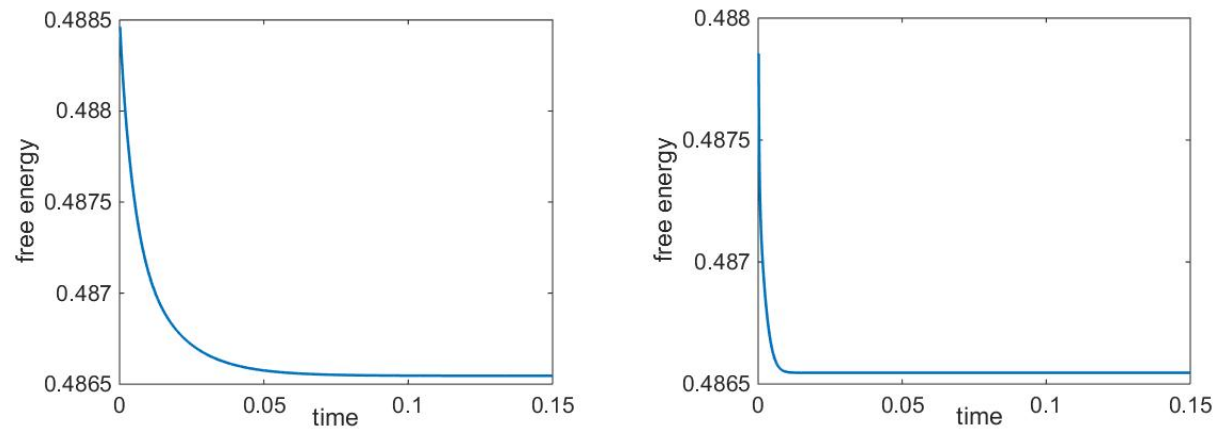

Figure 5. Evolution of the free energy along time, for $\Lambda_{x x}=$ $1, \Lambda_{y y}=1$ (on the left) and $\Lambda_{x x}=1, \Lambda_{y y}=100$ (on the right).

\section{Conclusion}

We proposed and analyzed a nonlinear energy stable scheme for solving the Richards equation. Moreover, the definition of the scheme only rely on physical quantities and not on artificial ones like for instance the Kirchhoff transform. We were able to carry out a full convergence analysis based on compactness arguments. Contrarily to classical schemes, this new nonlinear scheme produces no undershoot. As far as we know, our scheme is the first one to ensure that the discrete solution remains in the physical range even in the case of strong anisotropy.

However, it appears in the numerical simulations that in the case of strong anisotropy ratio, the scheme introduces an excessive numerical diffusion that makes its convergence very slow. This shall motivate the design of some new more robust schemes (for instance based on [10]) that preserve the main advantages of the scheme studied in this paper, namely the formulation in physical variables, the preservation of the physical range, and then control of the physical energy.

\section{Appendix A. Appendix}

\section{A.1. Some inequalities of Sobolev's type.}

Lemma A.1. Let $q \geq 1$, and let $u \in W^{1, q}(\Omega)$ be such that

$$
u \geq 0 \text { and } \lambda(\{u=0\}) \geq \alpha>0,
$$

where $\lambda$ denotes the 2 -dimensional Lebesgue measure. Define $q^{\star}=2 q /(2-q)$ if $q<2$ and $q^{\star}=+\infty$ if $q \geq 2$, then, for all $r \leq q^{\star}$ if $q \neq 2$ and $r<\infty$ if $q=2$, there exists $C$ depending only on $\Omega, r$, and $\alpha$ such that

$$
\|u\|_{L^{r}(\Omega)} \leq C\|\nabla u\|_{L^{q}(\Omega)^{2}} .
$$

Proof. Define the mean $\langle u\rangle$ value of $u$ by

$$
\langle u\rangle=\frac{1}{\lambda(\Omega)} \int_{\Omega} u(\boldsymbol{x}) \mathrm{d} \boldsymbol{x} \geq 0 .
$$

Due to the properties (73) of $u$, one has

$$
\int_{\Omega}|u-\langle u\rangle| \mathrm{d} \boldsymbol{x}=\int_{\{u=0\}}\langle u\rangle \mathrm{d} \boldsymbol{x}+\int_{\{u>0\}}|u-\langle u\rangle| \mathrm{d} \boldsymbol{x} \geq \alpha\langle u\rangle .
$$


On the other hand, thanks to Poincaré's inequality (see, e.g., [1]), one has

$$
\int_{\Omega}|u-\langle u\rangle| \mathrm{d} \boldsymbol{x} \leq \frac{\operatorname{diam}(\Omega)}{2} \int_{\Omega}|\nabla u| \mathrm{d} \boldsymbol{x} \leq \frac{\operatorname{diam}(\Omega)}{2} \lambda(\Omega)^{\frac{q-1}{q}}\|\nabla u\|_{L^{q}(\Omega)} .
$$

Therefore, we get that

$$
\langle u\rangle \leq \frac{\operatorname{diam}(\Omega)}{2 \alpha} \lambda(\Omega)^{\frac{q-1}{q}}\|\nabla u\|_{L^{q}(\Omega)} .
$$

Combining this estimate with Sobolev's inequality (see, e.g., [2]) yields

$$
\|u\|_{L^{r}(\Omega)} \leq\|u-\langle u\rangle\|_{L^{r}(\Omega)}+\lambda(\Omega)\langle u\rangle \leq C\|\nabla u\|_{L^{q}(\Omega)^{d}}
$$

where $C$ depends only the prescribed quantities.

In the next Lemma, we prove a discrete Sobolev inequality. Note that the proof takes advantage of the existence of a conformal $V_{\mathcal{T}}$, leading to a much simpler proof than in [16] or [6].

Lemma A.2. Let $\mathcal{T}$ and $\mathcal{M}$ be a primal and a dual discretizations of $\Omega$ as prescribed in \$2.1.1. Let $\left(u_{K}\right)_{K \in \mathcal{V}}$ be an arbitrary element of $\mathbb{R}^{\# \mathcal{V}}$, and denote by

$$
\left\langle u_{\mathcal{M}}\right\rangle=\frac{1}{\lambda(\Omega)} \int_{\Omega} u_{\mathcal{M}} \mathrm{d} \boldsymbol{x}=\frac{1}{\lambda(\Omega)} \int_{\Omega} u_{\mathcal{T}} \mathrm{d} \boldsymbol{x} .
$$

Then there exists $C$ depending only on $r, q \Omega$, and $\theta_{\mathcal{T}}$ such that

$$
\left\|u_{\mathcal{M}}-\left\langle u_{\mathcal{M}}\right\rangle\right\|_{L^{r}(\Omega)} \leq C \int_{\Omega}\left|\nabla u_{\mathcal{T}}\right|^{q} \mathrm{~d} \boldsymbol{x}, \quad \forall r \in[1, \infty), \forall q \geq \min \left(1, \frac{2 r}{2+r}\right) .
$$

Proof. Since $u_{\mathcal{T}}$ is Lipschitz continuous, the classical Sobolev inequality (cf. [2]) gives that

$$
\left\|u_{\mathcal{T}}-\left\langle u_{\mathcal{M}}\right\rangle\right\|_{L^{r}(\Omega)} \leq C \int_{\Omega}\left|\nabla u_{\mathcal{T}}\right|^{q} \mathrm{~d} \boldsymbol{x}, \quad \forall r \in[1, \infty), \forall q \geq \min \left(1, \frac{2 r}{2+r}\right) .
$$

It only remains to use (13) to conclude the proof.

With that discrete Sobolev inequality at hand (cf. Lemma A.2), we can now easily adapt the proof of Lemma A.1 to the discrete setting, leading to the following statement, whose proof is left to the reader.

Lemma A.3. Let $\left(v_{K}\right)_{K \in \mathcal{V}}$, and let $v_{\mathcal{M}}$ and $v_{\mathcal{T}}$ the corresponding discrete functions belonging to $X_{\mathcal{M}}$ and $V_{\mathcal{T}}$ respectively. Assume that

$$
v_{\mathcal{M}} \geq 0 \quad \text { and } \quad \lambda_{d}\left(\left\{v_{\mathcal{M}}=0\right\}\right) \geq \alpha>0,
$$

Define $q^{\star}=q d /(d-q)$ if $q<d$ and $q^{\star}=+\infty$ if $q \geq d$, then, for all finite $r \leq q^{\star}$, there exists $C$ depending only on $\Omega, \theta_{\mathcal{T}}, r$, and $\alpha$ such that

$$
\left\|v_{\mathcal{M}}\right\|_{L^{r}(\Omega)} \leq C\left\|\nabla v_{\mathcal{T}}\right\|_{L^{q}(\Omega)^{d}} .
$$




\section{A.2. Uniqueness of the weak solution.}

Proposition A.4. Under Assumptions (A1)-(A4), there exists a unique weak solution to the problem (1) in the sense of Definition 1.2.

Proof. First, define the full Kirchhoff transform $\varphi: \mathbb{R} \rightarrow \mathbb{R}$ by

$$
\varphi(p)=\int_{0}^{p} \eta(s(a)) \mathrm{d} a, \quad \forall p \in \mathbb{R} .
$$

It follows from Assumptions (A1) and (A2) that $\varphi$ is Lipschitz continuous, increasing, and fulfills $p \varphi(p)>0$ for all $p \neq 0$. Since $\eta$ is assumed to be bounded, one has

$$
\nabla \varphi(p)=\sqrt{\eta(s(p))} \nabla \xi(p) \in L^{2}\left(Q_{t_{f}}\right)^{d}
$$

for any $p: Q_{t_{f}} \rightarrow \mathbb{R}$ such that $\xi(p) \in L^{2}\left((0, T) ; H^{1}(\Omega)\right.$ ) (thus in particular for any weak solution). Therefore, any weak solution $p$ satisfies

$$
\iint_{Q_{t_{f}}} s(p) \partial_{t} \psi \mathrm{d} \boldsymbol{x} \mathrm{d} t+\int_{\Omega} s_{0} \psi(\cdot, 0) \mathrm{d} \boldsymbol{x}+\iint_{Q_{t_{f}}}(\eta(s(p)) \mathbf{g}-\nabla \varphi(p)) \cdot \Lambda \nabla \psi \mathrm{d} \boldsymbol{x} \mathrm{d} t=0
$$

for all $\psi \in C_{c}^{\infty}\left(\bar{\Omega} \times\left[0, t_{f}\right)\right)$. Mimicking Otto's uniqueness proof for degenerate parabolic-elliptic problems [26], we obtain that, given two weak solutions $p$ and $\widehat{p}$ corresponding to the same initial data $s_{0}$, one has

$$
\int_{\Omega}|s(p(\boldsymbol{x}, t))-s(\widehat{p}(\boldsymbol{x}, t))| \mathrm{d} \boldsymbol{x} \leq 0 \quad \text { for a.e. } t \geq 0,
$$

hence $s(p)=s(\widehat{p})$. Moreover, the mass being conserved, it follows from Assumption (A4) that

$$
0<\int_{\Omega} s(p(\boldsymbol{x}, t)) \mathrm{d} \boldsymbol{x}=\int_{\Omega} s_{0}(\boldsymbol{x}) \mathrm{d} \boldsymbol{x}=\bar{s}_{0} \operatorname{meas}(\Omega)<\operatorname{meas}(\Omega) \quad \text { for a.e. } t \geq 0 .
$$

Therefore,

$$
\mathcal{U}(t):=\operatorname{meas}\{\boldsymbol{x} \in \Omega \mid s(p(\cdot, t))<1\} \geq\left(1-\bar{s}_{0}\right) \operatorname{meas}(\Omega)>0 \quad \text { for a.e. } t \geq 0 .
$$

Since $s$ is increasing on $\left[p_{\star}, 0\right]$, one gets that $p(\cdot, t)=\widehat{p}(\cdot, t)$ on $\mathcal{U}(t)$ for a.e. $t \geq 0$.

Subtracting the weak formulation (74) corresponding to $p$ to the one for $\widehat{p}$ then yields

$$
\iint_{Q_{t_{f}}} \nabla(\varphi(p)-\varphi(\widehat{p})) \cdot \Lambda \nabla \psi \mathrm{d} \boldsymbol{x} \mathrm{d} t=0, \quad \forall \psi \in C_{c}^{\infty}\left(\bar{\Omega} \times\left[0, t_{f}\right)\right),
$$

and thus for all $\psi$ in $L^{2}\left((0, T) ; H^{1}(\Omega)\right)$ thanks to a density argument. Choosing $\psi=\varphi(p)-\varphi(\widehat{p})$ and using Assumption (A3) yields

$$
\|\nabla(\varphi(p(\cdot, t))-\varphi(\widehat{p}(\cdot, t)))\|_{L^{2}(\Omega)^{d}}=0 \quad \text { for a.e. } t \geq 0
$$

The function $\varphi(p)-\varphi(\widehat{p})$ is identically equal to 0 on $\mathcal{U}(t)$, we can apply Lemma A.1 to infer that

$$
\|\varphi(p(\cdot, t))-\varphi(\widehat{p}(\cdot, t))\|_{L^{2}(\Omega)}=0 \quad \text { for a.e. } t \geq 0
$$

Since $\varphi$ is increasing, one obtains that $p=\widehat{p}$ a.e. in $Q_{t_{f}}$. 


\section{REFERENCES}

[1] G. Acosta and R. G. Durán. An optimal Poincaré inequality in $L^{1}$ for convex domains. Proc. Amer. Math. Soc., 132(1):195-202 (electronic), 2004.

[2] R. A. Adams and J. J. F. Fournier. Sobolev spaces. Academic press (Elsevier), 2nd edition, 2003.

[3] H. W. Alt and S. Luckhaus. Quasilinear elliptic-parabolic differential equations. Math. Z., 183(3):311-341, 1983.

[4] B. Andreianov, C. Cancès, and A. Moussa. A nonlinear time compactness result and applications to discretization of degenerate parabolic-elliptic PDEs. working paper or preprint, 2015.

[5] T. Arbogast, M. F. Wheeler, and N.-Y. Zhang. A nonlinear mixed finite element method for a degenerate parabolic equation arising in flow in porous media. SIAM J. Numer. Anal., 33(4):1669-1687, 1996.

[6] M. Bessemoulin-Chatard, C. Chainais-Hillairet, and F. Filbet. On discrete functional inequalities for some finite volume schemes. IMA J. Numer. Anal., 35:1125-1149, 2015.

[7] K. Brenner, D. Hilhorst, and H. C. Vu Do. A gradient scheme for the discretization of Richards equation. In Finite volumes for complex applications. VII. Elliptic, parabolic and hyperbolic problems, volume 78 of Springer Proc. Math. Stat., pages 537-545. Springer, Cham, 2014.

[8] C. Cancès and C. Guichard. Entropy-diminishing CVFE scheme for solving anisotropic degenerate diffusion equations. In Finite volumes for complex applications. VII. Methods and theoretical aspects, volume 77 of Springer Proc. Math. Stat., pages 187-196. Springer, Cham, 2014.

[9] C. Cancès and C. Guichard. Convergence of a nonlinear entropy diminishing control volume finite element scheme for solving anisotropic degenerate parabolic equations. Math. Comp., 85(298):549-580, 2016.

[10] C. Cancès and C. Guichard. Numerical analysis of a robust free energy-diminishing Finite Volume scheme for degenerate parabolic equations with gradient structure. preprint HAL: hal-01119735, to appear in Found. Comput. Math., 2016.

[11] C. Cancès and M. Pierre. An existence result for multidimensional immiscible two-phase flows with discontinuous capillary pressure field. SIAM J. Math. Anal., 44(2):966-992, 2012.

[12] K. Deimling. Nonlinear functional analysis. Springer-Verlag, Berlin, 1985.

[13] J. Droniou. Finite volume schemes for diffusion equations: introduction to and review of modern methods. Math. Models Methods Appl. Sci., 24(8):1575-1619, 2014.

[14] R. Eymard, T. Gallouët, C. Guichard, R. Herbin, and R. Masson. TP or not TP, that is the question. Comput. Geosci., 18:285-296, 2014.

[15] R. Eymard, T. Gallouët, and R. Herbin. Finite volume methods. In Handbook of numerical analysis, Vol. VII, Handb. Numer. Anal., VII, pages 713-1020. North-Holland, Amsterdam, 2000.

[16] R. Eymard, T. Gallouët, and R. Herbin. Discretization of heterogeneous and anisotropic diffusion problems on general nonconforming meshes SUSHI: a scheme using stabilization and hybrid interfaces. IMA J. Numer. Anal., 30(4):1009-1043, 2010.

[17] R. Eymard, T. Gallouët, R. Herbin, M. Gutnic, and D. Hilhorst. Approximation by the finite volume method of an elliptic-parabolic equation arising in environmental studies. Math. Models Methods Appl. Sci., 11(09):1505-1528, 2001.

[18] R. Eymard, M. Gutnic, and D. Hilhorst. The finite volume method for Richards equation. Comput. Geosci., 3(3-4):259-294 (2000), 1999.

[19] P. A. Forsyth. A control volume finite element approach to NAPL groundwater contamination. SIAM J. Sci. Statist. Comput., 12(5):1029-1057, 1991.

[20] P. A. Forsyth and M. C. Kropinski. Monotonicity considerations for saturated-unsaturated subsurface flow. SIAM J. Sci. Comput., 18(5):1328-1354, 1997.

[21] T. Gallouët and J.-C. Latché. Compactness of discrete approximate solutions to parabolic PDEs-application to a turbulence model. Commun. Pure Appl. Anal., 11(6):2371-2391, 2012.

[22] R. Herbin and F. Hubert. Benchmark on discretization schemes for anisotropic diffusion problems on general grids. In Finite volumes for complex applications $V$, pages 659-692. ISTE, London, 2008. 
[23] R. Jordan, D. Kinderlehrer, and F. Otto. Free energy and the Fokker-Planck equation. Phys. $D, 107(2-4): 265-271,1997$. Landscape paradigms in physics and biology (Los Alamos, NM, 1996).

[24] R. A. Klausen, F. A. Radu, and G. T. Eigestad. Convergence of MPFA on triangulations and for Richards' equation. Int. J. Numer. Meth. Fl., 58(12):1327-1351, 2008.

[25] J. Leray and J. Schauder. Topologie et équations fonctionnelles. Ann. Sci. École Norm. Sup. (3), 51:45-78, 1934.

[26] F. Otto. $L^{1}$-contraction and uniqueness for quasilinear elliptic-parabolic equations. J. Differential Equations, 131:20-38, 1996.

[27] F. Radu, I. S. Pop, and P. Knabner. Order of convergence estimates for an euler implicit, mixed finite element discretization of richards' equation. SIAM J. Numer. Anal., 42(4):1452$1478,2004$.

[28] L. A. Richards. Capillary conduction of liquids through porous mediums. Journal of Applied Physics, 1(5):318-333, 1931.

[29] J. Simon. Compact sets in the space $L^{p}(0, T ; B)$. Ann. Mat. Pura Appl. (4), 146:65-96, 1987.

[30] R. L. Zarba, E. T. Bouloutas, and M. Celia. General mass-conservative numerical solution for the unsaturated flow equation. Water Resour. Res., 26(7):1483-1496, 1990.

\author{
Ahmed Ait Hammou Oulhaj and Claire Chainais \\ Laboratoire Paul Painlevé, UMR CNRS 8524 \\ Université Lille 1 \\ 59655 Villeneuve d'Ascq Cedex \\ ahmed.ait-hammou-oulhaj@math.univ-lille1.fr \\ Claire.Chainais@math. univ-lille1.fr \\ Clément CANCÈs \\ Team Rapsodi INRIA Lille - Nord Europe, \\ 40, avenue Halley, 59650 Villeneuve d'Ascq, France. \\ clement.cances@inria.fr
}

